\title{
Lattice and optics options for possible energy upgrades of the Large Hadron Collider
}

\author{
J. Keintzel, ${ }^{1,2, *}$ R. Tomás, ${ }^{1}$ R. Bruce, ${ }^{1}$ M. Giovannozzi $\odot,{ }^{1}$ T. Risselada, ${ }^{1}$ and F. Zimmermann ${ }^{1}$ \\ ${ }^{1}$ Beams Department, CERN, Esplanade des Particules 1, 1211 Geneva 23, Switzerland \\ ${ }^{2}$ TU Wien, Atominstitut, Stadionallee 2, 1020 Vienna, Austria
}

(Received 18 August 2020; accepted 5 October 2020; published 21 October 2020)

\begin{abstract}
Studies of possible energy upgrades of the Large Hadron Collider (LHC) aim at increasing the current nominal beam energy of $7 \mathrm{TeV}$ in view of expanding the discovery potential and physics reach of the LHC. Some critical aspects of the feasibility of partial or full energy upgrades are studied here, together with novel mitigation measures. Higher beam energies can be realized by pushing the installed main dipoles to their limits or by replacing different fractions of installed magnets. Moreover, a revised lattice design for a full energy upgrade, also known as the high-energy LHC (HE-LHC), is presented. The studies reported focus on the linear optics design and on the lattice layout. In particular, an optics with $60^{\circ}$ transverse phase advance in the regular arc cells, meant to alleviate the strength requirements for the main quadrupoles, has been designed in view of possible beam tests during the next LHC run 3. The methods developed in this article, for matching the ring geometry to a reference tunnel, for improving the beam aperture, and for minimizing the required field strengths for new high-energy LHC-like machines, will also be applicable to other future circular collider projects.
\end{abstract}

DOI: 10.1103/PhysRevAccelBeams.23.101602

\section{INTRODUCTION AND MOTIVATION}

The Large Hadron Collider (LHC) [1] at CERN is a synchrotron of almost $27 \mathrm{~km}$ circumference, where two counterrotating proton beams, with a design beam energy of $7 \mathrm{TeV}$, collide at four interaction points (IPs), dedicated to high-energy particle physics experiments. In previous runs a beam energy up to $6.5 \mathrm{TeV}$ has been achieved.

The LHC's luminosity upgrade, the high luminosity LHC (HL-LHC) [2], is scheduled to be commissioned around 2027 and to operate through the late 2030s. With a nominal beam energy of $7 \mathrm{TeV}$, a leveled luminosity of at least $5 \times 10^{34} \mathrm{~cm}^{-2} \mathrm{~s}^{-1}$ is envisioned at the two primary interaction points (IPs) 1 and 5, where the ATLAS and CMS experiments are located. One key ingredient of HL-LHC is the limited, first-ever use of $\mathrm{Nb}_{3} \mathrm{Sn}$ magnet technology in a storage-ring collider. Namely, 16 inner triplet $\mathrm{Nb}$-Ti quadrupoles around the two primary collision points will be replaced by larger-aperture $\mathrm{Nb}_{3} \mathrm{Sn}$ quadrupoles, to allow for the desired stronger focusing (smaller $\beta^{*}$ ), and a couple of $11 \mathrm{~T}$ dipoles will be installed in the dispersion suppressors of one collimation interaction

\footnotetext{
*jacqueline.keintzel@cern.ch
}

Published by the American Physical Society under the terms of the Creative Commons Attribution 4.0 International license. Further distribution of this work must maintain attribution to the author(s) and the published article's title, journal citation, and DOI. region, to make space for extra collimators in this critical region of the machine.

Possible hadron-collider successors of the HL-LHC are currently being explored within the frame of the future circular collider (FCC) study [3,4]. The FCC hadron collider (FCC-hh) [5] is designed to reach a beam energy of about $50 \mathrm{TeV}$, making use of thousands of $16 \mathrm{~T} \mathrm{Nb}_{3} \mathrm{Sn}$ main dipoles. With a circumference of about $100 \mathrm{~km}$, for the FCC, a whole new infrastructure will need to be built and connected with the existing CERN accelerator complex. The FCC integrated project [6] foresees as a first stage the electron-positron collider FCC-ee [7] - serving as a Higgs and electroweak factory-similar to the former large electron positron collider (LEP) [8] preceding the present LHC. The results presented in this article, focusing on the integration of new higher-energy LHC-like machines in an existing tunnel infrastructure, might also naturally be applicable to the FCC integrated project [6], or to a similar pair of lepton and hadron colliders proposed in China (CEPC, SppC) [9]. For example, the various approaches described could help match the footprints of the FCC-ee and FCC-hh colliders, maximize the FCC-hh aperture at injection, and minimize the FCC-hh magnet field strengths.

In addition to the FCC, the so-called high-energy LHC (HE-LHC) [10,11] has also been studied. Its present baseline design assumes the installation, in the existing LHC tunnel, of new dipoles based on the FCC-hh magnet technology. The HE-LHC design aims at reaching 13.5 TeV beam energy. The HE-LHC would be installed 
TABLE I. Nominal parameters for (HL-)LHC, HE-LHC and FCC-hh for proton operation.

\begin{tabular}{lccc}
\hline \hline Parameter & (HL-)LHC & HE-LHC & FCC-hh \\
\hline Circumference $[\mathrm{km}]$ & 27 & 27 & 100 \\
Beam energy [TeV] & $(7) 7$ & 13.5 & 50 \\
Arc dipole field [T] & $(8.33) 8.33$ & 16 & 16 \\
Protons per bunch [10 & $(2.2) 1.15$ & 2.2 & 1.0 \\
Normalized rms emittance $\gamma \varepsilon_{x, y}[\mu \mathrm{m}]$ & $(2.5) 3.75$ & 2.5 & 2.2 \\
IP $\beta$-function $\beta_{x, y}^{*}[\mathrm{~m}]$ & $(0.15) 0.55$ & 0.45 & 0.3 \\
Half crossing angle $[\mu \mathrm{rad}]$ & $(250) 142.5$ & 165 & 100 \\
Peak luminosity $\mathcal{L}_{\max }\left[10^{34} \mathrm{~cm}^{-2} \mathrm{~s}^{-1}\right]$ & $(5$, levelled) 1 & 16 & 30 \\
Annual luminosity $\mathcal{L}_{\text {int }}\left[\mathrm{fb}^{-1} \mathrm{yr}^{-1}\right]$ & $(>250) 55$ & 500 & 1000 \\
\hline \hline
\end{tabular}

in the tunnel infrastructure that already hosted LHC and, earlier, LEP. Furthermore, the HE-LHC design assumes the replacement of most of the presently installed HL-LHC elements. Key parameters of LHC, HL-LHC, HE-LHC, and FCC-hh $[1,2,5,11,12]$ are given in Table I.

Increasing the beam energy of the current HL-LHC baseline could enlarge the discovery potential [13]. For this reason, various options for reaching beam energies above $7 \mathrm{TeV}$, determined mainly by the reachable main dipole field, have already previously been considered [14-16]. In this paper, we present new, comprehensive, and detailed optics developments for various approaches towards a higher-energy LHC. Options for increasing the reachable beam energy include pushing the field of the installed main dipoles to the ultimate value of $9 \mathrm{~T}$, as already considered in $[1,14,15]$, as well as replacing different fractions of main dipoles by stronger ones with fields up to $16 \mathrm{~T}[15,16]$. These partial energy upgrades would aim at increasing the beam energy while limiting the number of new magnets, thus reusing most of the existing elements. Compared to previous studies [15-17], which focused primarily on replacing $1 / 3$ of installed main dipoles by $11 \mathrm{~T}$ magnets $[15,16]$ or replacing the existing $\mathrm{Nb}-\mathrm{Ti}$ arc quadrupoles by $\mathrm{Nb}_{3}$ Sn combined-function magnets [15], the scope of our studies is enlarged. This paper also considers replacing higher fractions of dipoles with new ones, whose magnetic field spans the entire range from 11 to $16 \mathrm{~T}$. Although higher energies are, to first order, made possible by higher bending fields, the limit imposed by the required quadrupolar gradients should not be neglected. In fact, the present $90^{\circ}$ phase-advance optics of HL-LHC is limited to a beam energy of about $8.6 \mathrm{TeV}$, as main quadrupoles would exceed their limits. A reduced phase-advance optics of $60^{\circ}$ could alleviate this limit. For a similar reason, that is, to reduce the strengths of arc quadrupoles and sextupoles, a $60^{\circ}$ arc-cell phase advance was previously proposed for the HE-LHC [18].

Intriguingly, for the former design of the Superconducting Super Collider (SSC), the exactly opposite optics change was made [19]: to facilitate the dispersion suppressors (DSs), in 1987 the betatron phase advance per arc cell was increased from $60^{\circ}$ in the SSC Conceptual Design Report [20] to $90^{\circ}$
$[19,21]$. Coincidentally, in the same year 1987, two designs featuring different cell phase advances $\left(60^{\circ}\right.$ and $\left.90^{\circ}\right)$ were also studied for the LHC. Constraints from the LEP layout had been incorporated in these proposed designs: A $60^{\circ}$ cell option had been conceived with a length of $79 \mathrm{~m}$ [8] equal to the LEP arc cell, whereas for the alternative $90^{\circ}$ layout the cell length had almost been doubled compared with LEP, to $156 \mathrm{~m}$ [22]. For either case, the arc-cell lengths had been chosen so as to facilitate the integration of the LHC on top of LEP in the same tunnel [22]. Subsequent studies of both layouts revealed the advantage, e.g., in terms of dynamic aperture, when including the expected sextupolar field errors in the superconducting dipole magnets, of a $90^{\circ}$ option with a maximum arc-cell length of $120 \mathrm{~m}$ [23]. Retracting the requirement for the LHC arc-cell length to be a multiple of the LEP one, and following the inclusion of higher-order magnetic field errors in the tracking studies later led to a further reduction of the arc-cell length to about $100 \mathrm{~m}$, while maintaining a phase advance of $90^{\circ}[24,25]$. The current LHC design consisting of $23106.9 \mathrm{~m}$-long arc cells with $90^{\circ}$ phase advance was first published in 1995 [26].

In the following, we present a detailed analysis of a $60^{\circ}$ optics for a partial energy upgrade of today's LHC, with emphasis on the DS.

A more radical approach for an LHC energy upgrade consists in reusing only the tunnel infrastructure and redesigning entirely the accelerator ring, based on novel magnet technology. This is the approach pursued by the HE-LHC design [10,11]. An overall baseline has already been presented in the HE-LHC conceptual design report [11]. However, for one of the two optics baseline designs presented in [11] the peak-to-peak offset from the LEP tunnel geometry exceeded the one of the LHC by about $5 \mathrm{~cm}$ with collimators in the DS ( $1 \mathrm{~cm}$ without collimators), while the other baseline option did not allow for the installation of any DS collimators. In this paper, we report significant improvements of both baseline HE-LHC layouts [11], including a further optimized configuration for the arcs with particular attention to the DS geometry, thus allowing the possibility to install DS collimators for improved collimation efficiency as in HL-LHC [2], while keeping the offset from the LEP tunnel geometry within 
acceptable bounds, i.e., within less than $5 \mathrm{~cm}$ from the LEP tunnel. In addition to these essential improvements of the previous designs [11], we discuss various options to increase the beam aperture, including an alternative HELHC optics based on combined-function dipoles and a new beam screen design, optimized for maximum beam aperture. While the existing HE-LHC baseline design with $90^{\circ}$ arc-cell phase advance is improved to better fulfill all the imposed constraints, an alternative arc-cell phase-advance optics with $60^{\circ}$ (first proposed in [18]) is also studied, in analogy to the aforementioned study for pushing the energy of the present LHC. Interestingly, for HE-LHC the $60^{\circ}$ optics allows for a higher dipole filling factor and hence opens up a higher energy reach. Furthermore, the $60^{\circ}$ arccell phase-advance optics could help suppress the effect of magnet nonlinearities [18].

The present article focuses on linear optics, layout optimization, and performance reaches. It does not address nonlinear beam dynamics resulting from magnetic imperfections or emittance growth from magnetic flux jumps in the $\mathrm{Nb}_{3} \mathrm{Sn}$ superconductor [27]. However, the required level of field errors determined in past studies can be found in the various LHC, HL-LHC and HE-LHC design reports $[1,2,11]$. The figures would need to be scaled with the arc optics, e.g., linearly in average $\beta$ function for the transverse instability growth rates, and with the power $1 / \beta^{n / 2}$ for field error tolerances, where $n=2$ refers to a quadrupole error, etc. Also, collective effects are not discussed here. Resistive wall impedance for the reduced, and possibly varying, beam-wall distance and collimator wakefields for tighter physical gap sizes would need to be examined.

We will proceed as follows. Section II presents a brief introduction to the LHC and the HL-LHC lattice, followed by a discussion of lattice and optics constraints in Sec. III. Possible partial energy upgrade options are discussed in Sec. IV. Section V presents the latest developments of the HE-LHC layout. A $60^{\circ} \mathrm{LHC}$ optics is proposed in Sec. VI. This can be considered as a general-purpose quadrupole strength mitigation option for high-energy colliders, including the HE-LHC [18]. Finally, some conclusions are drawn in Sec. VII.

\section{LAYOUT OF LHC AND HL-LHC MACHINES}

Figure 1 [28] presents the identical conceptual layout of LHC and HL-LHC. Both accelerators consist of eight periodic arcs, each containing 23 arc cells with a FODO structure (periodic structure of focusing quadrupole, drift space, defocusing quadrupole and drift space), connecting eight insertion regions (IRs). One (HL-)LHC FODO cell, schematically shown in Fig. 2, contains six main dipoles (MBs), equipped with a sextupole spool piece corrector (MCS). After every second MB, octupole (MCO) and decapole (MCD) spool piece correctors are placed. The short straight section (SSS), i.e., the bending-free region, is made of a beam position monitor (BPM), a trim quadrupole

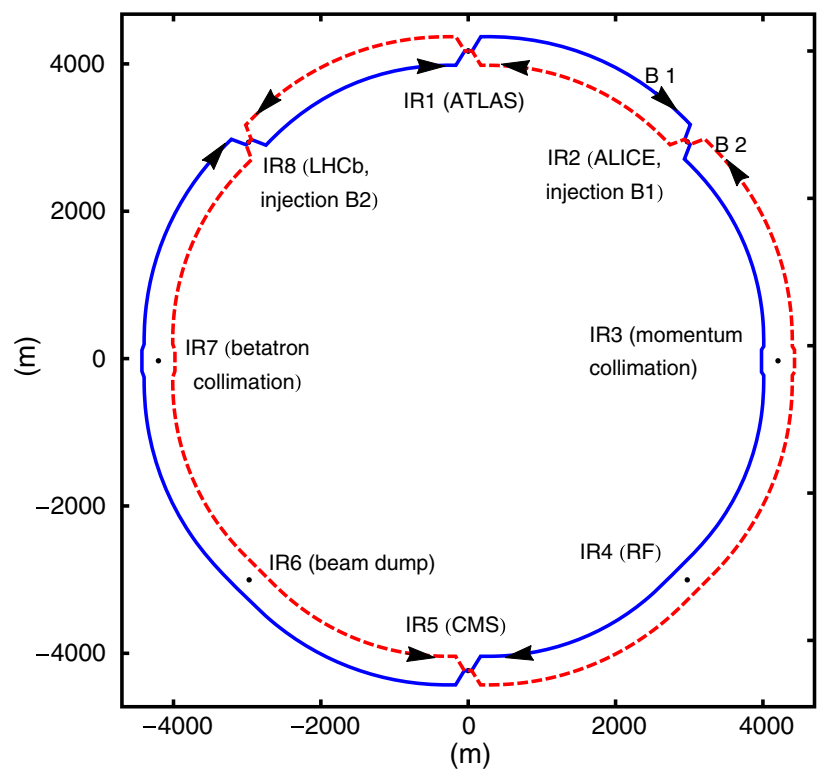

FIG. 1. Schematic layout of the (HL-)LHC (from [28]). Beam 1 (B1) and beam 2 (B2) are shown, respectively, in solid blue and dashed red. The beam separation is not to scale.

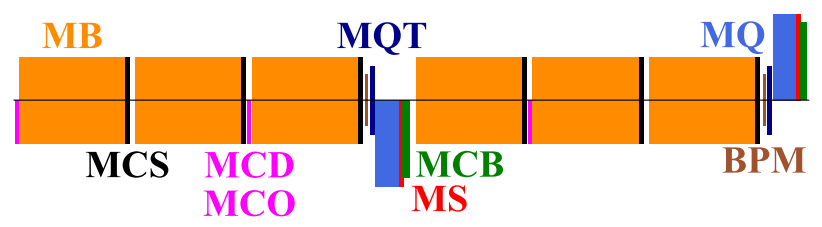

FIG. 2. Schematic plot of a LHC FODO cell, consisting of six main dipoles (MBs), each equipped with a sextupole spool piece corrector (MCS). An octupole (MCO) and a decapole (MCD) corrector are placed after every second MB. The short straight section is equipped with a main quadrupole (MQ), a trim quadrupole (MQT), an orbit corrector (MCB), a main sextupole (MS) and a beam position monitor (BPM). Focusing and defocusing quadrupoles, respectively, are shown below and above the horizontal axis.

(MQT), a main quadrupole (MQ), a main sextupole (MS) and a dipolar orbit corrector (MCB). In every arc eight of the MQTs are replaced by four octupoles (MO) and four skew quadrupoles (MQS), and four of the MS per arc by skew sextupoles (MSS). The MSS are used for the correction of the chromatic coupling [29-31].

The general-purpose ATLAS and CMS experiments are located in IRs 1 and 5. IR2 and IR8 house the ALICE and $\mathrm{LHCb}$ experiments, together with the beam 1 and beam 2 injection systems. Momentum and betatron cleaning are installed in IR3 and IR7, respectively. The radio frequency (rf) insertion is located in IR4, where also the transverse fast bunch-by-bunch feedback system and parts of the LHC beam instrumentation are located. The beam extraction is in IR6, from where the LHC beams are ejected through a $600 \mathrm{~m}$ long beam dump transfer line onto a beam dump 


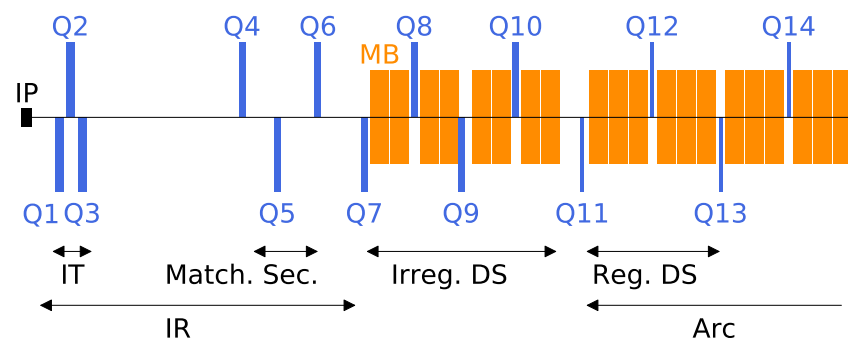

FIG. 3. Schematic layout of quadrupoles (Q) and main dipoles (MBs) of the lattice from the IP to Q14, for an experimental IR, where the latter spans from the IP to the start of Q7. Q1 to Q3 form the inner triplet (IT). The interaction region (IR) proper extends through Q7. After the matching section (Q4 to Q6), the irregular part of the dispersion suppressor (DS) spans from Q7 to Q11. Following a drift space the regular DS includes Q11 to Q13, and the regular arc lattice structure starts at Q11.

block. Optics-wise, all insertions feature a maximum of seven quadrupole magnets on each side of the interaction point, where in IRs without beam crossing the term "IP" simply marks the center of the straight section. In the experimental IRs, a triplet, so-called inner triplet (IT), made of three optical quadrupoles, Q1, Q2 and Q3, is installed on each side of the IP. It has to be noted that Q2 is split into two physical quadrupole magnets, Q2a and Q2b. In other IRs, where the beams do not cross, no triplets are installed. The elements of the "outer triplet," Q4 to Q6, where all of them exist, are used for matching and have individual power supplies.

To match the periodic arc optics to the dedicated IR one, dispersion suppressors (DSs) are installed in the transition region, where the DSs is strictly speaking separated in two parts. The first part, in the following also referred to as irregular part, is located after the matching section of the IR and spans from individually powered Q7 to Q10, where between two quadrupole magnets only two dipoles are placed, contrarily to three dipoles in regular FODO cells, and, therefore, represents a missing dipole dispersion suppressor scheme. This first part of the DS is then followed by a drift space of about $13 \mathrm{~m}$. The second part of the DS, in the following called the regular part, starts with the first periodic arc FODO cell and extends from Q11 to Q13, where individually powered trim quadrupoles are installed, close to the main quadrupoles MQ11, MQ12, and MQ13. This lattice structure, from the IP up to Q14, is schematically shown in Fig. 3 for an experimental IR. Note that for the HL-LHC the DSs on both sides of IR7 will be modified by replacing one MB by two $11 \mathrm{~T}, 5.307 \mathrm{~m}$ long $\mathrm{Nb}_{3} \mathrm{Sn}$ dipoles with a collimator (TCLD) in between. The regular arc optics starts after Q13 and spans over 21 FODO cells.

\section{LATTICE AND OPTICS CONSTRAINTS}

The energy upgrade options are based on the HL-LHC design. Therefore, the conceptual layout including the arc
FODO-cell structure, the location of the experiments, beam injection, collimation, beam dump and rf sections remain unchanged. Generating new lattices demands respecting minimum element-to-element distances, which are different for partial energy upgrade options or the full upgrade, respectively. For the first case, HL-LHC drift spaces are kept to simplify the design study, while for the latter, HELHC drift spaces are preserved; their lengths were adopted from the FCC-hh design. A summary of minimum drift spaces between various elements is given in Table II.

Beam energy, and hence the magnetic rigidity $B \rho$ of a synchrotron is determined by the length of the main bending magnets $L_{\mathrm{MB}}$, their bending angle $\theta$, bending radius $\rho$ and the magnetic field $B$ through [32]

$$
B \rho=\frac{B L_{\mathrm{MB}}}{\theta}, \quad \theta=\frac{L_{\mathrm{MB}}}{\rho} .
$$

Currently, the LHC is operated at $6.5 \mathrm{TeV}$ beam energy, which is achieved with a field of $7.74 \mathrm{~T}$ in the 1232 main dipoles, whereas the nominal energy of $7 \mathrm{TeV}$ requires the nominal field of $8.33 \mathrm{~T}$ [1]. These $\mathrm{Nb}$-Ti dipoles are bent to match the particle path and have an interbeam distance of $194 \mathrm{~mm}$. In the following considerations about partial energy upgrades, the installed LHC dipoles will be called weak dipoles; they were designed to reach the ultimate field of $9.0 \mathrm{~T}[1]$.

In various $R \& D$ programs $[5,33,34]$ dipoles with design field up to $16 \mathrm{~T}$ are studied. For possible partial energy upgrades, the use of dipoles generating a field in the interval 11 to $16 \mathrm{~T}$ is assumed, with the same interbeam distance of $194 \mathrm{~mm}$ as for the present LHC. These magnets are called strong dipoles in the following. The HE-LHC, however, foresees the use of $16 \mathrm{~T}$ dipoles similar to those designed for FCC-hh [5], with an interbeam distance of $250 \mathrm{~mm}$, which is required to assure the needed field quality at injection.

The installed (HL-)LHC arc quadrupoles provide a nominal gradient of $223 \mathrm{Tm}^{-1}$ at $7 \mathrm{TeV}$ beam energy, which is required for a phase advance $\mu$ per FODO cell in both transverse planes $x, y$ of about $90^{\circ}$. It has to be noted that $\mu_{x, y}$ of one FODO cell is not exactly $90^{\circ}$ in the LHC. For the HL-LHC optics, exactly $90^{\circ}$ phase advance is kept

TABLE II. Minimum required element spacing for HL-LHC and HE-LHC layouts.

\begin{tabular}{llc}
\hline \hline Drift between elements [m] & HL-LHC & HE-LHC \\
\hline MB-MB & 1.36 & 1.5 \\
MB-BPM & 1.153 & 1.3 \\
BPM-MQT & 0.591 & 0.35 \\
MQT-MQ & 0.301 & 0.35 \\
MQ-MS & 0.1605 & 0.35 \\
MS-MCB & 0.085 & 0.35 \\
MCB-MB & 1.1035 & 1.3 \\
\hline \hline
\end{tabular}


in the arcs adjacent to the high-luminosity experiments, since this is required for implementing the achromatic telescopic squeezing (ATS) optics [35]. A gradient of $278 \mathrm{Tm}^{-1}$ [36] is assumed to be the maximum achievable, and hence arc quadrupoles are limited at a beam energy of 8.7 $\mathrm{TeV}$ with $90^{\circ}$ optics. One possibility to lower the required integrated quadrupole gradient $K$, while keeping the cell length $L_{\text {cell }}$ unchanged, is choosing a design with smaller phase advance $\mu$ as $[18,32]$

$$
\frac{1}{K}=\frac{L_{\text {cell }}}{4 \sin (\mu / 2)} \text {. }
$$

In the present LHC, different quadrupole types with specific strength constraints are used for Q1 to Q10 for each IR, where further information can be found in [1]. For HELHC, the quadrupole strength limit is set to $360 \mathrm{Tm}^{-1}$ for Q1 to Q7 [11]. It has to be noted that the inner triplet gradient is well below this limit, and resulted from a tradeoff between aperture, magnet length, and radiation load [37]. Furthermore, the minimum gradient at injection energy must be greater than $3 \%$ of the nominal one at maximum beam energy [1]. This constraint is related to the accuracy of the power converters.

Possible upgrades of the HL-LHC need to fit into the existing tunnel infrastructure and need, therefore, to have a similar footprint as HL-LHC, LHC or LEP. The geometry offset between a new lattice and a reference lattice, LEP or LHC, refers to the radial difference of two lattices sharing the same center. This radial offset, $r_{2}-r_{1}$, is calculated for about 5000 different angles $\alpha$, spanned from the center of the ring, with $0 \leq \alpha<2 \pi$, by

$$
r_{2}-r_{1}=\sqrt{x_{2}^{2}+y_{2}^{2}}-\sqrt{x_{1}^{2}+y_{1}^{2}},
$$

where the suffixes 1 and 2 are used for the reference lattice and a new lattice, respectively. This offset concept is schematically shown in Fig. 4. In this context, the peakto-peak distance of (HL-)LHC to LEP of about $6.5 \mathrm{~cm}$ must, therefore, not be exceeded significantly for future configurations and represents an essential constraint on the overall ring layout.

During the LHC design phase and later, the evaluation of the beam stay clear (BSC), needed to ensure protection of the superconducting magnets from beam losses, was an essential task. In this article, the BSC at any given longitudinal location is defined as the minimum transverse distance between the beam center and the mechanical aperture, expressed in units of the local transverse beam size and including tolerances for various sources of imperfections. It is evaluated through a numeric algorithm [38], implemented in the program MAD-X [39], to find the smallest distance, normalized by the local transverse beam size, between the closed orbit and the physical aperture in the transverse plane. In the computation, error tolerances

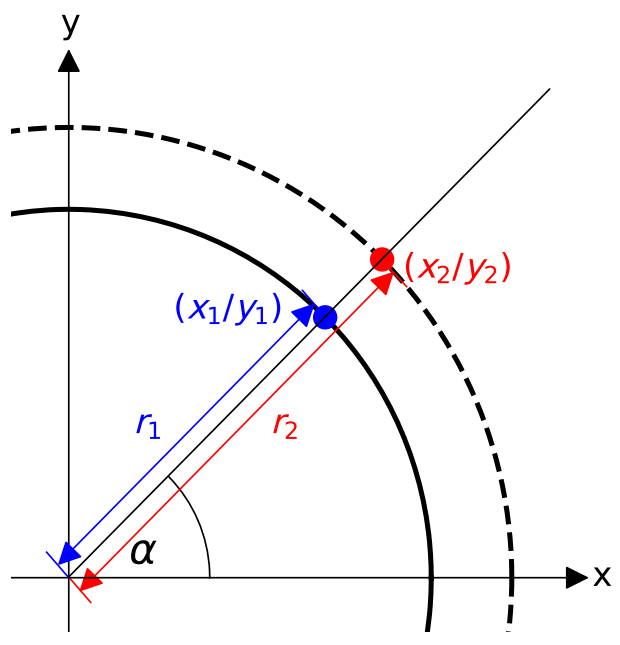

FIG. 4. Schematic plot of radial difference between two concentric circular accelerators, approximated by circles, at a given angle $\alpha$.

are applied to the aperture itself, as well as to the orbit and optics. For the computations in this article, the mechanical tolerances of typically $1 \mathrm{~mm}$ (installation and alignment, ground motion, beam screen tolerances) are assumed to be equal to those of the LHC [40]. The beam tolerances are assumed to be the same as for HL-LHC [41-43], where we most notably assume a $2 \mathrm{~mm}$ closed-orbit radial tolerance, a relative momentum offset of $8.6 \times 10^{-4}$ and a $10 \% \beta$ beating at the $450 \mathrm{GeV}$ injection energy, as well as a fractional parasitic dispersion from the arc of $14 \%{ }^{1}$

The computed BSC around the ring has to be compared to the minimum acceptable BSC above which it can be considered safe [38,41-43]. The determination of the acceptable BSC involves the analysis of the performance of the collimation system that is supposed to intercept and dispose of high-amplitude particles [1,45-47]. For HLLHC at injection, the minimum BSC has been computed to $12.6 \sigma$ based on studies of the halo distribution in various loss scenarios [42]. For possible future energy upgrades, in this paper the BSC goal, at an injection energy of $450 \mathrm{GeV}$, is tentatively set to $10 \sigma$. This tighter limit assumes increased quench limits of new magnets, improved tolerances, such as better controlled closed orbit and smaller optics beating, and the possibility to operate with tighter collimator settings that ensure protection of aperture bottlenecks also in failure scenarios, as in [48].

While the geometrical properties of the beam screen of the arc and DS magnets define the BSC at injection energy, the beam screens of the IR magnets define the BSC at top energy and in collision configuration with the $\beta^{*}$ squeezed to its smallest value. In the studies presented here, the arc

\footnotetext{
${ }^{1}$ The parasitic dispersion is the fraction of the horizontal peak arc dispersion, scaled by the ratio $\sqrt{\beta_{\mathrm{IR}} / \beta_{\text {arc }}}$, which remains in the IRs after the optics correction [44].
} 


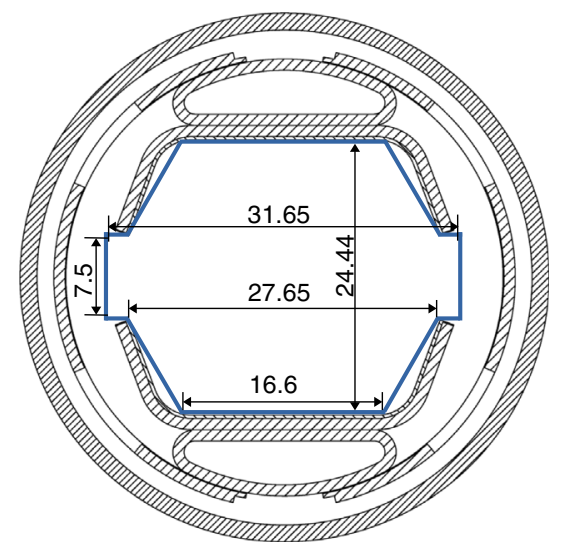

FIG. 5. Cross section of FCC-hh beam screen, adapted from [49], values are given in $\mathrm{mm}$. The blue line displays the implementation in MAD-X.

and DS beam screens of LHC and FCC-hh are assumed for all existing and new elements in DSs and arcs, respectively, where the FCC-hh design is shown in Fig. 5 [49]. We note that the location and, especially, the transverse dimensions, of the helium cooling channels for the LHC and the FCChh arc beam screens differ substantially. A detailed engineering design would be required, taking into account constraints from the cryogenics and vacuum systems, in order to ensure the feasibility of the assumed hybrid configuration, or to determine the needed technical modifications to connect the two types of beam screens. The present LHC magnets in the IRs feature again different beam screens (more details can be found in $[1,11]$ ).

LHC main dipoles are bent to follow the beam path, thus optimizing the BSC. In a pessimistic scenario, the new dipoles might be straight leading to a beam sagitta ( $\mathrm{sag}$ ) in the dipoles of

$$
\operatorname{sag} / 2=\rho \sin ^{2} \frac{\theta}{4}
$$

Figure 6 shows the reference beam paths through bent and straight dipoles, including sag/2, which, for a LHC-type $14 \mathrm{~m}$ long bending magnet, is of order $4 \mathrm{~mm}$, compared to half of the available horizontal half aperture of about $14 \mathrm{~mm}$ (Fig. 5). Note that in addition to aperture loss, this effect is equivalent to an orbit offset, where possible feed-down effects from the magnetic field quality of the
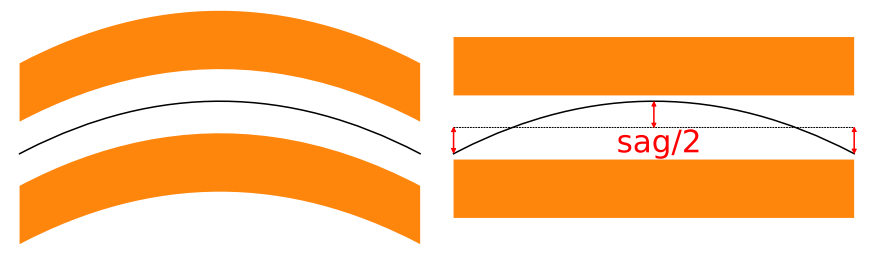

FIG. 6. Reference beam path through bent (left) and straight (right) dipoles and resulting beam sagitta (sag). dipoles might not be completely negligible. However, these effects are outside of the scope of the studies discussed in this paper.

\section{PARTIAL ENERGY UPGRADE OPTIONS}

All possible partial energy upgrades of the HL-LHC rely on increasing the effective dipole strength, which can be realized by increasing the global integrated field of the MBs [15-17] or by replacing the arc MQ by combined-function dipoles [15]. In the following we study the first option, which can be realized either by pushing the installed MBs to their maximum field of $9 \mathrm{~T}$ or by replacing different fractions of existing dipoles by stronger ones, with a field up to $16 \mathrm{~T}$.

\section{A. Pushing all installed dipoles}

If all existing main dipoles can successfully be pushed to $9 \mathrm{~T}$, a beam energy of about $7.5 \mathrm{TeV}$ is attained. This option was already foreseen in the LHC design report [1]. This field would require dedicated training with about 2.8 quenches expected per dipole [16], but it would allow reusing the vast majority, of installed components, although not all of the magnetic elements, with a $90^{\circ}$ optics [16]. The full implications of pushing the ring to ultimate beam energy were recently reviewed, and a detailed analysis can be found in [16]. This $8 \%$ energy upgrade with respect to the nominal HL-LHC does not require any new dipoles. Therefore, it implies the present BSC of about $13.4 \sigma$ at $450 \mathrm{GeV}$ in the arcs. The footprint of the lattice also remains unchanged from (HL-)LHC.

\section{B. Partial replacement of main dipoles}

Partial replacement of main dipoles, upgrading one-third ( $1 / 3$ option) or two-thirds (2/3 option) of the arc MBs is studied (complementing the studies presented in [17]), while preserving minimum HL-LHC drift spaces from Table II. The energy reach for various partial energy upgrade options assumes strong main $\mathrm{Nb}_{3} \mathrm{Sn}$ dipoles, generating strong $\mathrm{MB}$ fields ranging from 11 to $16 \mathrm{~T}$, and weak Nb-Ti dipoles, generating fields of 7.74, 8.33, or $9 \mathrm{~T}$. The beam-energy reach for various combinations of weak and strong dipole fields is shown in Fig. 7, together with a theoretical full upgrade, which assumed LHC element lengths and minimum-drift spaces between them, however, with strong MB fields of up to $16 \mathrm{~T}$. It is assumed that in the case of intermediate energy upgrade options, weak and strong MBs in the arcs are part of the same powering circuit, in order to ease their integration.

As expected, beam energy increases linearly with increasing dipole fields. For example, replacing $1 / 3$ of the arc dipoles with $16 \mathrm{~T}$ ones and pushing existing ones to $9 \mathrm{~T}$ leads to a beam energy of $9.53 \mathrm{TeV}$, whereas for the corresponding $2 / 3$ option $11.50 \mathrm{TeV}$ can be achieved. In general, $2 / 3$ options lead to $10 \%$ to $20 \%$ higher beam 


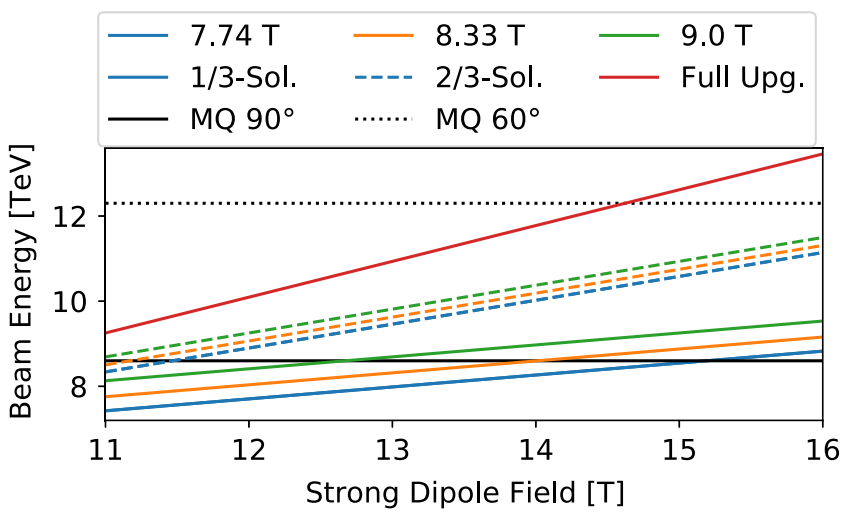

FIG. 7. Reachable beam energy for various $1 / 3$ solutions (solid lines), 2/3 solutions (dashed lines), assuming $7.74 \mathrm{~T}$ (blue), $8.33 \mathrm{~T}$ (orange), or $9 \mathrm{~T}$ (green) weak dipole fields, together with a full upgrade (red). The maximum beam energy compatible with the main quadrupoles limits is 8.6 and $12.3 \mathrm{TeV}$ for a $90^{\circ}$ and a $60^{\circ}$ arc-cell phase-advance design, respectively.

energy as the corresponding $1 / 3$ option, assuming the same weak and strong dipole fields. Moreover, pushing installed dipoles to ultimate field increases the beam energy by about $2 \%$ or $5 \%$ for $2 / 3$ or $1 / 3$ options, respectively. It has to be noted that several other magnets, such as separation dipoles and insertion quadrupoles, should be replaced too [17].

In addition to the difference in strength, the two dipole types could also differ in their geometry: the weak, existing ones are bent to follow the LHC geometry. Here, we pessimistically assume that the strong ones are straight as for the FCC-hh, since a bending of $\mathrm{Nb}_{3} \mathrm{Sn}$ dipoles has not yet been demonstrated, and that they are equipped with the FCC-hh beam screen. The combination of unequal dipole strength, dipole straightness, and the small aperture of the FCC-hh beam screen significantly reduces the available minimum physical aperture in the horizontal plane, compared with the present LHC dipoles, and also compared with the FCC-hh.

The lowest BSC of about $4.3 \sigma$ in the arcs at $450 \mathrm{GeV}$ is found for $2 / 3$ solution with strong, $16 \mathrm{~T}$, and weak, $7.74 \mathrm{~T}$, dipoles, which is significantly below the limit of $10 \sigma$ and is hence assumed to be too low to operate efficiently the

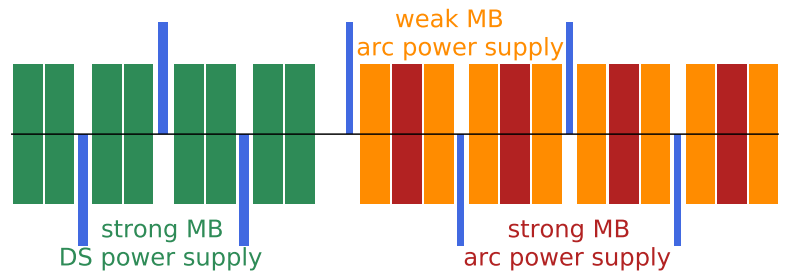

machine. However, using the LHC beam screen also for strong dipoles would increase this minimum BSC in the arcs to about $10 \sigma$. Note that although the installed weak main dipoles are bent, their curvature is no longer optimized for partial upgrade options, leading to a difference between beam and magnet sagitta inside these dipoles, due to the different field strengths of weak and strong magnets. The resulting orbit offset of about $1 \mathrm{~mm}$ from the center of the LHC dipoles and the resulting BSC reduction is, however, negligible. The use of two different strengths for dipoles in FODO cells changes the bending radius with respect to the HL-LHC DS, leading to a massive geometry offset with respect to LHC of over $1 \mathrm{~m}$ peak-to-peak, if only weak dipoles are used in the DS. To restore a more reasonable geometry, dipole fields in the DS need to be adjusted, assuming the same DS lattice as in HL-LHC. Installing new main dipoles in all DSs with a bending angle per dipole $\left(\theta_{\mathrm{DS}}\right)$ equal to the average bending strength per half FODO cell $\left(\theta_{\mathrm{hc}}\right)$,

$$
\theta_{\mathrm{DS}}=\left\langle\theta_{\mathrm{hc}}\right\rangle,
$$

results in the best possible geometry offset with respect to LHC, which means that $\left\langle\theta_{\mathrm{DS}}\right\rangle$ must stay the same as in HLLHC in each DS.

For example, for a $1 / 3$ option with $7.74 \mathrm{~T}$ weak, and $16 \mathrm{~T}$ strong dipoles, a field of $10.5 \mathrm{~T}$ in the DS is demanded. These DS dipoles are assumed to be of the same type as the strong dipoles in the arcs, although they would need to be connected to a different power supply, as schematically shown in Fig. 8 (left) for the DS right of the IRs. With this configuration a geometry offset below $1 \mathrm{~cm}$ peak-to-peak in the arcs and $0 \mathrm{~cm}$ in the DS is achieved, as shown in Fig. 9 (left). This DS and the arc configuration demand the installation of $496(40 \%)$ or $864(70 \%)$ new main dipoles for the $1 / 3$ or $2 / 3$ solution, respectively.

Another possibility is to replace only half of the DS dipole magnets [50], as shown in Fig. 8 (right) for a $1 / 3$ lattice. In this scheme, the new DS dipoles would also need to be fed by a separate power supply, different from the arc dipoles. In case of a lattice with $7.74 \mathrm{~T}$ weak and $16 \mathrm{~T}$ strong dipoles, MBs in the DS require a field of $13.3 \mathrm{~T}$.

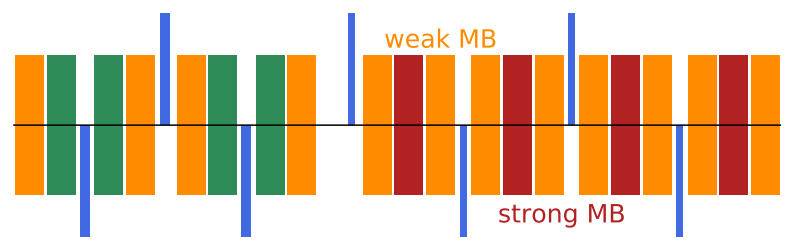

FIG. 8. Left: main dipoles, quadrupoles and powering scheme of the DSs and the arcs for a 1/3 option resulting in a peak-to-peak of $1 \mathrm{~cm}$ and a constant offset below $1 \mathrm{~cm}$ in the arcs and DSs, respectively. Strong and weak MBs are shown in red and orange, respectively. MBs in the DS, shown in green, are the same type as strong dipoles in the arcs, but have a different power supply. Right: a different geometry and powering scheme for the $1 / 3$ option resulting in a peak-to-peak of $1 \mathrm{~cm}$ and below $2 \mathrm{~cm}$ in the arcs and DSs, respectively. 

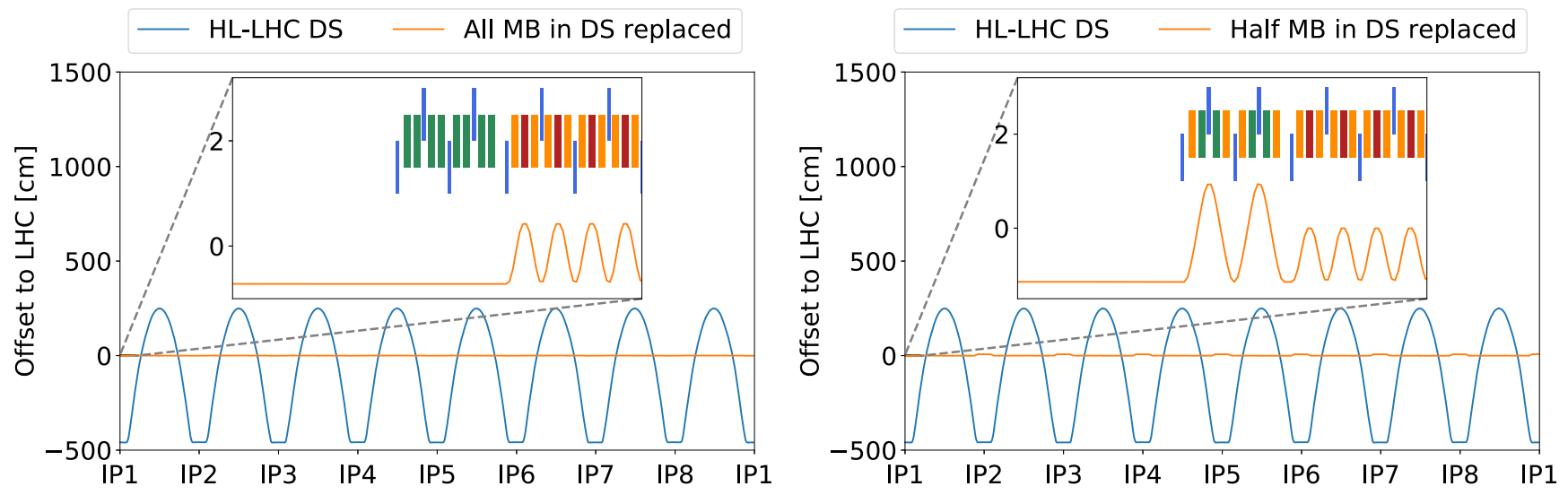

FIG. 9. Left: impact of nonoptimized (blue) and optimized (orange) DS bending radius on geometry offset with respect to LHC for a $1 / 3$ option with $7.74 \mathrm{~T}$ weak, and $16 \mathrm{~T}$ strong dipoles, by replacing all DSs dipoles, together with the lattice shown in Fig. 8 (left) and resulting in a geometry offset of $0 \mathrm{~cm}$ and below $1 \mathrm{~cm}$ peak-to-peak in the DSs and the arcs, respectively. Right: same, but for the lattice shown in Fig. 8 (right) and resulting in a geometry offset of about $2 \mathrm{~cm}$ and below $1 \mathrm{~cm}$ peak-to-peak in the DSs and the arcs, respectively.

This option increases the geometry offset in the DS to about $1.5 \mathrm{~cm}$ peak-to-peak, as is shown in Fig. 9 (right). This second solution for the DS lattice does not appear suitable for a 2/3 option, as the required DS MB field would exceed the strength of the strong dipoles in the arcs. Alternative DS solutions would need to be explored in future studies, if the integration of two different power supplies, one for the DS and one for the arcs, would not be feasible.

The highest reachable beam energy with a partial energy upgrade is $11.50 \mathrm{TeV}$, exceeding the maximum limit for main quadrupoles of $8.7 \mathrm{TeV}$ with a $90^{\circ}$ phase-advance optics. However, using an optics with $60^{\circ}$ (see Sec. VI and Fig. 7) can push the available strength of the main quadrupoles to $12 \mathrm{TeV}$, which allows reusing HL-LHC main quadrupoles and, therefore, helps limit the demand for new magnets.

\section{FULL ENERGY UPGRADE-THE HE-LHC}

The HE-LHC represents a full-energy upgrade of the HL-LHC, as almost all of the installed elements are assumed to be replaced. While respecting all minimum HE-LHC drift spaces, given in Table II, various arc cell and DS options have been generated and analyzed [51], converging on two baseline designs, namely an $18 \times 90$ (18 FODO cells per arc with $90^{\circ}$ phase advance), and a $23 \times 90$ [11,52] LHC-like lattice. These lattice designs are further optimized to overcome the challenges described in Sec. I. Complementary studies regarding impedance, collimation, dynamic aperture, and performance have been performed in [11,53-56].

\section{A. Improving lattice geometry}

FODO cell, MB, and DS lengths are optimized to reduce the geometry offset with respect to LEP while increasing the dipole filling factor (the fraction of the total cell length occupied by the main dipoles). For HE-LHC, LEP is chosen as a reference, as LHC has also been designed to minimize the geometry offset with respect to LEP. To simplify lattice generation and optimization, a software application named ALGEA (automatic lattice generation application) has been developed [57]. Based on a few input parameters such as minimum drift spaces, type of DS, and number of FODO cells, ALGEA generates LHC-like lattices, in a format compatible with MAD-X. It is found that the geometry offset with respect to LEP can be significantly reduced if the following parameters are properly adjusted.

Arc-cell length.-To minimize the arc offset of HE-LHC with respect to LHC, the effective FODO-cell bending radius $\rho_{\text {cell }}$ of the new lattices needs to be as close as possible to the one of LHC:

$$
\rho_{\text {cell }}^{\mathrm{HE}} \stackrel{!}{=} \rho_{\text {cell }}^{\mathrm{LHC}},
$$

where the effective bending radius for a FODO cell is defined as [also see Eq. (1)]

$$
\rho_{\text {cell }} \equiv \frac{L_{\text {cell }}}{N_{\mathrm{MB}} \theta},
$$

with $N_{\mathrm{MB}}$ denoting the number of MBs per FODO cell and $\theta$ the MB bending angle, defined by the total number of MBs. It is assumed that the center of MQs has no transverse offset with respect to the closed orbit generated by the MBs to avoid any feed-down effect. Optimizing the arc-cell length, therefore, reduces to a purely geometric challenge, depending only on MBs (number and length) and on the FODO-cell length. If the FODO cells of the new lattice are too long, a U-shaped footprint with respect to the reference lattice is observed as can be seen in Fig. 10 for a design with 18 FODO cells per arc. On the other hand, a too short FODO cell results in a footprint bent upwards. By choosing 


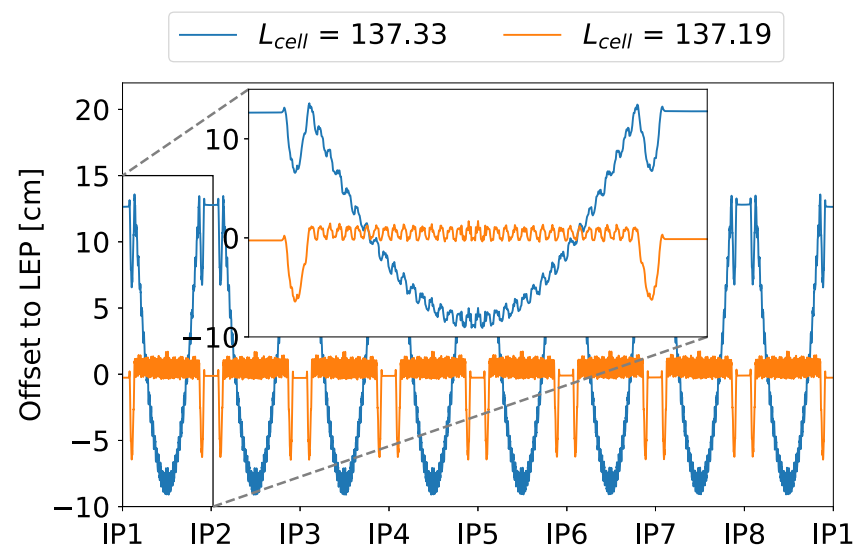

FIG. 10. Effect on the geometry offset with respect to LEP of choosing an optimized cell length of $137.19 \mathrm{~m}$ for an 18-cell HE-LHC design, compared with a cell length of $137.33 \mathrm{~m}$.

the correct cell length, the arc geometry offset is reduced to about $2 \mathrm{~cm}$ peak-to-peak.

Arc position.-The overall transverse arc offset can be controlled by adjusting the central position of the arc proper (composed of regular FODO cells) with respect to the unmoved irregular DS part on both sides of the arc. If all arcs are shifted counterclockwise by about $1.2 \mathrm{~m}$, as seen in Fig. 11 (left) for an 18-cell lattice, the footprint assumes a sinusoidal-like shape. Each arc is tilted with respect to the reference lattice, hence the radial offset varies from the start to the end of the arc. If arcs are aligned exactly in the middle between two irregular DS this leads to straightening of the footprint, since the reference footprint also is centered.

Dispersion suppressor position.-Even though the geometry offset in the IRs is assumed to be slightly less restricted than for the arcs and the DS, an offset as small as possible is aimed for in this design study. Geometry offset in the IRs can be reduced by adjusting the position of the irregular DS part with respect to the IP. For an 18-cell
HE-LHC design shifting all irregular DSs by $3 \mathrm{~m}$ towards the IR reduces the IR geometry offset by about $11 \mathrm{~cm}$ as shown in Fig. 11 (right).

\section{B. Improved arc optics}

Using these geometry-optimization techniques, the existing $23 \times 90$ and $18 \times 90$ lattice options have been revisited and optimized. The FODO cells feature the same elements as in LHC and are displayed in Fig. 12. Each main dipole is equipped with a sextupole spool piece corrector. In addition, octupole and decapole correctors are attached to every second dipole. The short straight section, i.e., the bending-free space between a dipole and a quadrupole, accommodates an orbit corrector, a sextupole, a beam position monitor, and a trim quadrupole. Similar to LHC, eight MQTs per arc are exchanged against four octupoles and four quadrupoles, and four MS per arc are replaced by skew sextupoles. HE-LHC FODO-cell parameters are given in Table III. The resulting FODO-cell lengths are 137.19 and $106.9 \mathrm{~m}$ for the $18 \times 90$ and the $23 \times 90$ designs, respectively. With a filling factor of $81 \%$ and $77 \%$, beam energy of 13.62 and $12.92 \mathrm{TeV}$ is reached using $16 \mathrm{~T}$ dipoles. Note that to reach $13.5 \mathrm{TeV}$, the $23 \times 90$ design would require $16.7 \mathrm{~T}$ dipoles. Table III reveals that the MQs are kept below their gradient limit of $360 \mathrm{Tm}^{-1}$, leaving a few percent of margin. Using exactly $360 \mathrm{Tm}^{-1}$ would allow shortening MQs and lengthening the MBs, thereby further increasing the dipole filling factor and the energy reach. However, in this case, the resulting additional improvement is negligible.

The longer cells of the $18 \times 90$ design require weaker gradients, thus making the main quadrupoles shorter, and increase the energy reach, although this leads to a smaller BSC. At $450 \mathrm{GeV}$ the two options reach $7.37 \sigma$ and $8.78 \sigma$, respectively, assuming the FCC-hh beam screen, shown in Fig. 5.
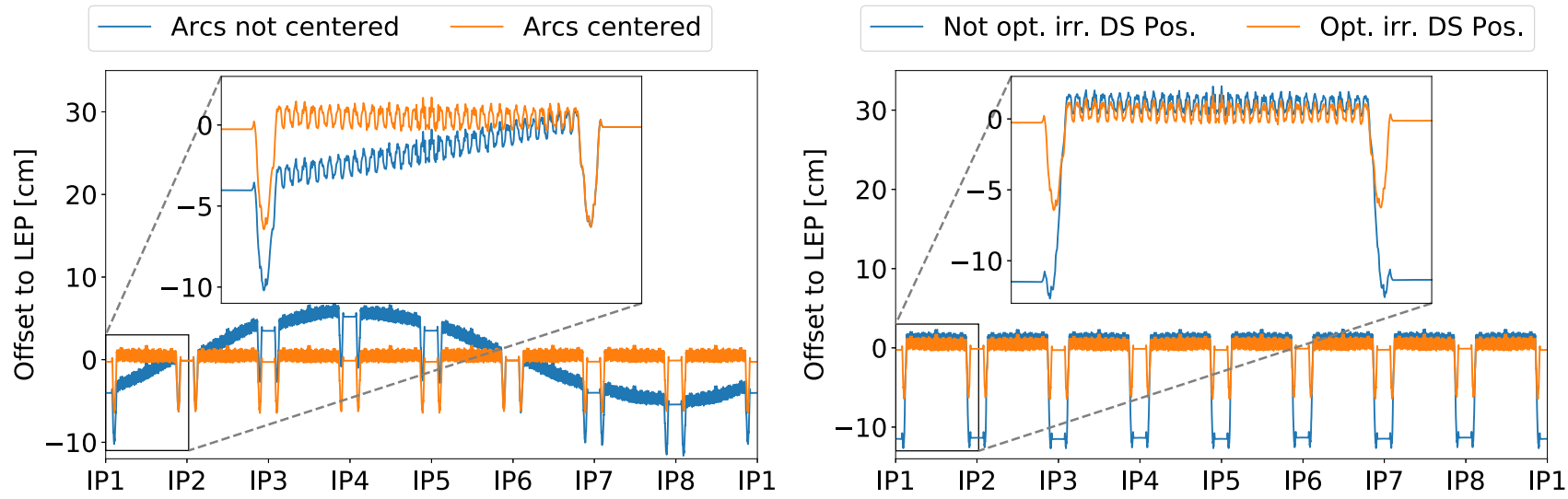

FIG. 11. Upper: Impact of shifting the arc position by $1.2 \mathrm{~m}$ counterclockwise for an 18-cell HE-LHC design on the geometry offset with respect to LEP. Lower: Impact of shifting DS position with respect to the interaction point. Shifting all DSs $3 \mathrm{~m}$ towards the IRs reduces the geometry offset in the interaction region by about $11 \mathrm{~cm}$ with respect to LEP for an 18-cells-per-arc HE-LHC design. 


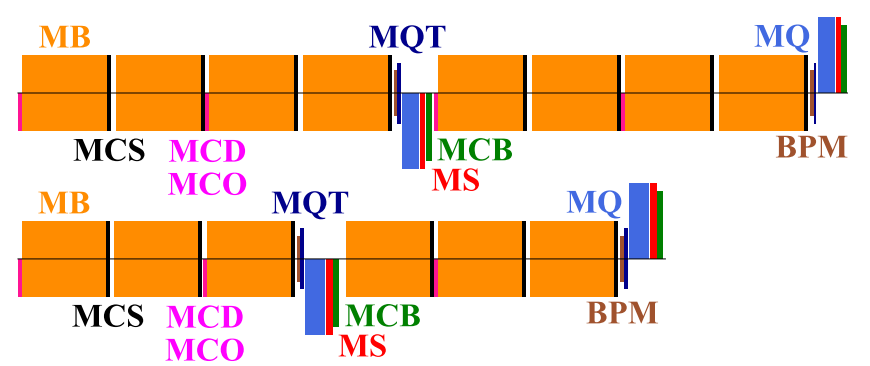

FIG. 12. Arc-cell layout of the $18 \times 90$ (upper) and $23 \times 90$ (lower) design. In both arc-cell designs, MBs are each equipped with a sextupole spool piece corrector (MCS). Similar as in LHC, an octupole (MCO) and a decapole (MCD) corrector are placed after every two MBs. Next to the main quadrupole (MQ), a trim quadrupole (MQT), an orbit corrector (MCB), a main sextupole (MS), and a beam position monitor (BPM) are located. Focusing and defocusing quadrupoles are represented by bars being located below and above the horizontal axis, respectively.

As one major design constraint is BSC, especially at injection energy, effort was put into finding mitigation measures in order to increase it so as to reach at least $10 \sigma$ for both designs. It has to be noted that changes in the cell phase advance did not result in any meaningful improvement of the BSC. Nevertheless, a proper adjustment of the phase advance [58] could allow resonance cancellations that improve the dynamic aperture, which could be explored in future studies. Various options to improve the BSC are discussed in detail in the following.

\section{Higher injection energy}

The present SPS injection energy of $450 \mathrm{GeV}$ is not sufficient to reach $10 \sigma$ BSC. An intermediate energy upgrade of the SPS, replacing about half of the SPS magnets by superconducting ones, could provide a beam energy of about $600 \mathrm{GeV}$ [59]. Within the framework of the HE-LHC design study, SPS upgrades resulting in $900 \mathrm{GeV}$

TABLE III. Parameters of the two HE-LHC design options, compared with the existing (HL-)LHC.

\begin{tabular}{|c|c|c|c|}
\hline \multirow[b]{2}{*}{ Collider Parameter } & \multicolumn{2}{|c|}{ HE-LHC } & \multirow{2}{*}{$\frac{\mathrm{LHC}}{23 \times 90}$} \\
\hline & $18 \times 90$ & $23 \times 90$ & \\
\hline Cell length $[\mathrm{m}]$ & 137.19 & 106.90 & 106.9 \\
\hline Dipoles per cell & 8 & 6 & 6 \\
\hline Dipole length [m] & 13.92 & 13.73 & 14.3 \\
\hline Filling factor $[\%]$ & 81 & 77 & 80 \\
\hline Quadrupole length [m] & 2.8 & 3.3 & 3.1 \\
\hline Quadrupole strength $\left[\mathrm{Tm}^{-1}\right]$ & 335 & 352 & 223 \\
\hline$\beta_{\min } / \beta_{\max }[\mathrm{m}]$ & $41 / 230$ & $32 / 177$ & $32 / 177$ \\
\hline$D_{\min } / D_{\max }[\mathrm{m}]$ & $1.7 / 3.6$ & $1.1 / 2.2$ & $1.0 / 2.0$ \\
\hline Dipole field $[\mathrm{T}]$ & 16 & 16 & 8.33 \\
\hline Beam energy $[\mathrm{TeV}]$ & 13.62 & 12.92 & 7 \\
\hline Field for $13.5 \mathrm{TeV}[\mathrm{T}]$ & 15.8 & 16.7 & $\cdots$ \\
\hline Arc BSC at $450 \mathrm{GeV}[\sigma]$ & 7.37 & 8.78 & 13.4 \\
\hline
\end{tabular}

or even $1.3 \mathrm{TeV}$ injection energy in the HE-LHC have been considered. In case of the $23 \times 90$ HE-LHC optics, $600 \mathrm{GeV}$ are sufficient to reach $10 \sigma \mathrm{BSC}$ and hence an intermediate SPS upgrade would be sufficient. On the other hand, the $18 \times 90$ design requires at least $830 \mathrm{GeV}$, thus imposing a full upgrade of the SPS.

Integration issues for a combination of warm and cold powering circuits, and the age of the existing warm SPS magnets, would both seem to favor a complete replacement of the SPS by a new superconducting machine.

It has to be noted that at higher energy, losses are more destructive and the quench limit is lower. Therefore, more margins are needed between the protection devices and the aperture, meaning that the minimum allowed BSC (in units of beam $\sigma$ ) is expected to be higher at a higher energy in order to ensure sufficient protection. The exact limit would need to be explored in further studies. On the other hand, the achieved BSC in any element is also expected to be higher, since the beam size is smaller. Assuming that this latter effect is stronger, as is the case in the LHC [43], it could be easier to reach the BSC target at a higher energy.

\section{Combined-function dipoles}

Combined function dipoles feature, in addition to a dipole field, also a quadrupole component $\left(b_{2}\right)$, hence providing additional focusing, thus decreasing the beam size and increasing BSC. The magnetic field expansion reads [44]

$$
B_{y}+i B_{x}=B_{\text {ref }}\left[1+b_{2}\left(\frac{x+i y}{R_{\text {ref }}}\right)\right],
$$

where $B_{\text {ref }}$ represents the dipolar magnetic field and $b_{2} B_{\text {ref }}$ the additional quadrupole field at the reference radius $R_{\text {ref }}$.

As the minimum BSC in a FODO cell is found at the defocusing quadrupole, the goal of this study is to determine additional $b_{2}$ components in the MBs that reduce $\beta_{y}$ at the defocusing quadrupole. To find the required quadrupolar component, $b_{2}$ is stepwise increased while testing all possible combinations of positive and negative quadrupolar fields in the MBs and keeping a transverse phase advance of $90^{\circ}$. The best result is obtained by adding $b_{2}$ components of $\pm 515 \times 10^{-4}$ and $\pm 500 \times 10^{-4}$ (with $R_{\text {ref }}=16.7 \mathrm{~mm}$ ), which reduces the peak vertical $\beta$ function to about $133 \mathrm{~m}$ $(-42 \%)$ and $138 \mathrm{~m}(-22 \%)$ for the $18 \times 90$ and the $23 \times 90$ FODO cell, respectively. In this way, the goal of $10 \sigma$ BSC can be reached.

Due to $b_{2}$ components in the MBs, quadrupole gradients are significantly decreased to about $36 \mathrm{Tm}^{-1}$ for the $18 \times 90$ option, and to about $240 \mathrm{Tm}^{-1}$ for the $23 \times 90$ one. The quadrupoles can, therefore, be made shorter; this frees additional space for the MBs and increases the filling factor to about $85 \%$ and $79 \%$. It has to be noted that this assumes the feasibility of two different dipole types, providing e.g., $+500 \times 10^{-4}$ on beam 1 and $-500 \times 10^{-4}$ on 


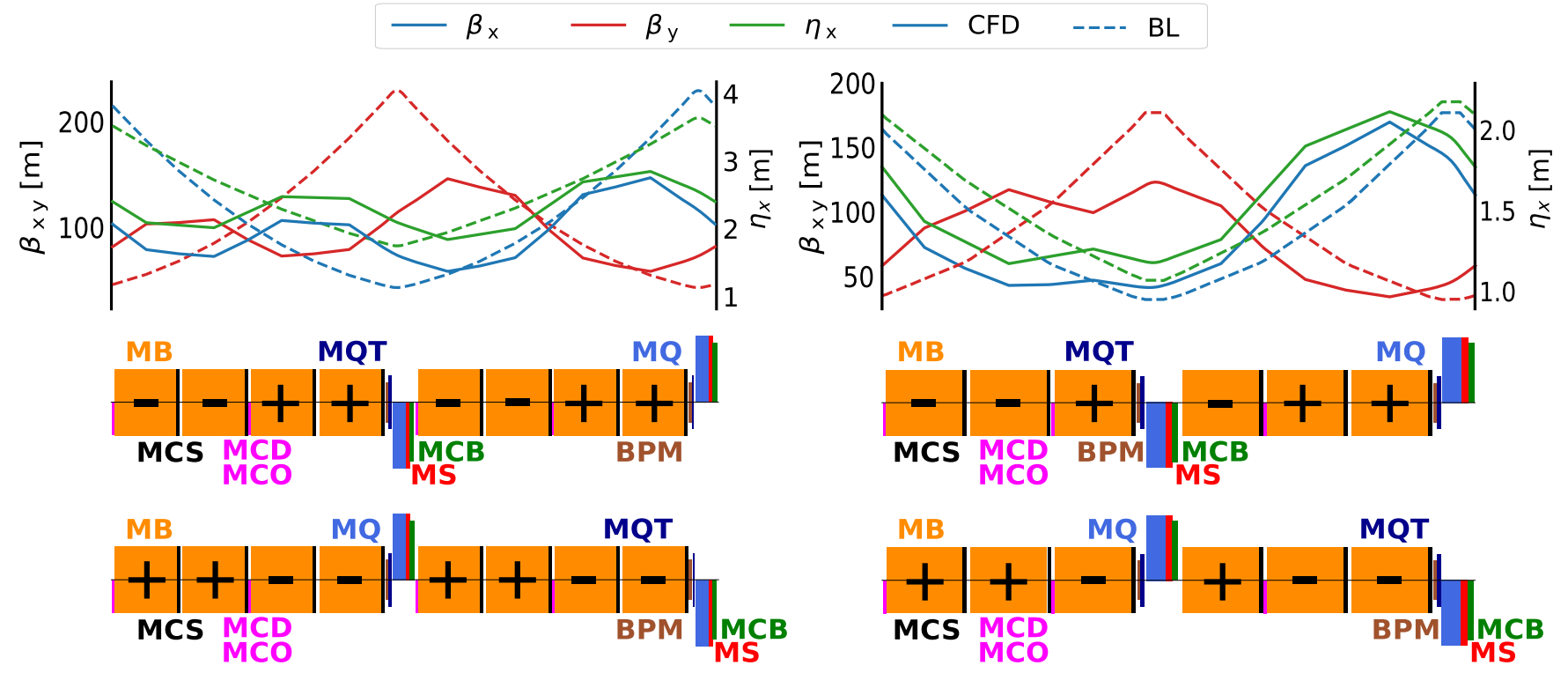

FIG. 13. $18 \times 90$ FODO-cell (left) and $23 \times 90$ (right) optics for the baseline (BL) cell and with the use of combined-function dipoles (CFD) for beam 1 (top) and the required quadrupole component (middle). For beam 2 only the required quadrupole components are shown (bottom). The plus and minus signs represent additional positive and negative quadrupole components of $\pm 515 \times 10^{-4}$ and $\pm 500 \times 10^{-4}$ units, respectively, for the $18 \times 90$ and the $23 \times 90$ optics.

beam 2 and vice versa. The resulting optics functions including required quadrupole components for the both layouts for beam 1 are shown in Fig. 13 (top and middle), together with the baseline design. The bottom plots in Fig. 13 show the required combination of additional $b_{2}$ for beam 2 .

\section{Optimized beam screen design}

As it has already been demonstrated during the design of the LHC, the choice of beam-screen geometry highly impacts the BSC [60]. The FCC-hh beam screen has been fully optimized for cooling efficiency, vacuum properties, impedance and electron-cloud suppression etc., but it is not necessarily the best choice for the aperture and optics optimization under discussion here. Note that by increasing the FCC-hh beam screen by a scaling factor of 1.10 or 1.22 for the $23 \times 90$ and $18 \times 90$, respectively, we may obtain a $10 \sigma$ BSC in the FODO cells. Nonetheless, a full optimization can be carried out to find the most suitable beam screen design for the HE-LHC, from the aperture point of view alone.

Improving the cross section of the beam screen can be achieved by computing the beam ellipses at all the locations of magnetic elements along the arc, up to the DS in IR5, and overlaying them. The beam ellipses are constructed using an equal emittance in the horizontal and vertical planes (as it is done for the evaluation of the BSC), whose dimensions equal the selected BSC. As far as the centers of the ellipses are concerned, these are obtained by combining the mechanical tolerances, orbit, and dispersion at the location of each magnetic element. Due to the symmetry of the beam screen, there are four beam ellipses for each location. Such a superposition of beam ellipses is seen in Fig. 14 for the $18 \times 90$ optics at $450 \mathrm{GeV}$ energy.

The current beam screen shape in Fig. 14 (left), where the minimum BSC of $7.6 \sigma$ using a relative momentum offset of $3.1 \times 10^{-4}$ has been selected to plot the beam ellipses, provides a poor balance between vertical and horizontal planes, with an inefficient use of the available transverse cross section area of the magnet inner coils volume. Therefore, without considering any implications for the vacuum system, beam screen cooling, or impedance, a different shape of the beam screen, much more efficient in terms of usage of cross section area, could be considered. Such aperture-optimized beam screen is displayed in
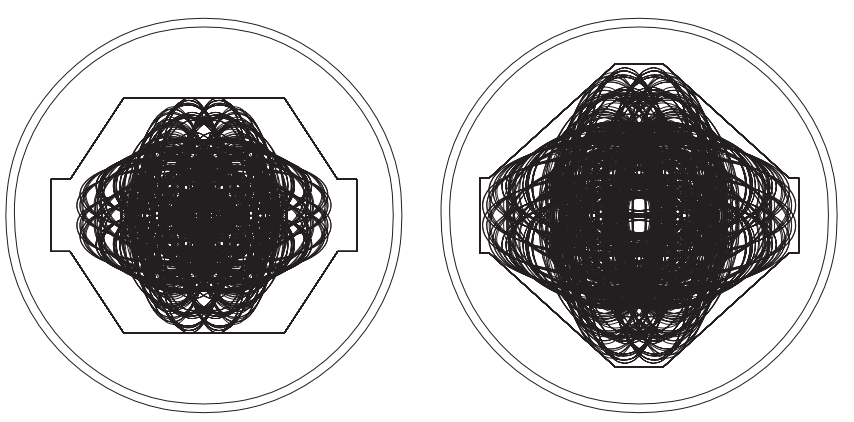

FIG. 14. FCC-hh beam screen (left) and the new design proposal (right) with beam ellipses superimposed. The beam ellipses are calculated for the magnetic elements in the regular arc cell and the IR5 DS, for the $18 \times 90$ cell layout at $450 \mathrm{GeV}$ beam energy and a relative momentum offset of $3.1 \times 10^{-4}$. The number of betatron sigmas used is equal to the corresponding minimum BSC value within the range of beam elements. 
Fig. 14 (right), where the BSC constraint is set to $10 \sigma$. This shape features increased horizontal dimension of about $31 \mathrm{~mm}$ and vertical dimension of about $30 \mathrm{~mm}$. It has to be noted that for the $23 \times 90$ optics, with a $10 \%$ larger BSC, the dimensions of the optimized beam screen could be made smaller by approximately the same amount.

It is worth noting that the optimized cross section of the beam screen seems to converge towards a shape that is close to a square, which is the shape proposed in [60] for the LHC, and close to an octagon, which is the shape selected for HL-LHC [2]. Indeed, the results of the numerical simulations indicate that the need of aperture in the horizontal direction should not be overemphasized for these high-energy rings, as the vertical motion also requires sufficient clearance.

\section{Improved aperture tolerances}

To estimate how the aperture tolerances impact the BSC figure of merit, scans over the values of these tolerances have been performed for both HE-LHC FODO-cell layouts using MAD-X. For these studies, the closed-orbit, $\beta$ beating, and parasitic dispersion uncertainty, as well as tolerances of the mechanical aperture itself have been considered. The relation between the variation of BSC as a function of the tolerances can be assumed to be linear within the used ranges, where the upper boundary is identical to the default aperture tolerances, described in Sec. III.

Setting these four tolerances to zero, i.e., identical to the lower boundary of the parameter space, would result in a BSC in the arc cells of approximately $11.1 \sigma$ and $12.6 \sigma$ for the $18 \times 90$ and the $23 \times 90$ lattice, respectively. Hence, in this case, both options would be compatible with the target $\mathrm{BSC}$ for the $450 \mathrm{GeV}$ injection energy.

Applied closed-orbit uncertainty of $2 \mathrm{~mm}$ is found to reduce the BSC significantly, i.e., by about $1.7 \sigma$ and $1.9 \sigma$, respectively, whereas an improved closed orbit tolerance of $1 \mathrm{~mm}$, which assumes that all orbit correctors are always available during beam operations [42], would increase the BSC to about $8.5 \sigma$ and $9.8 \sigma$, compared to the case of nominal aperture parameters, i.e., approximately $7.4 \sigma$ and $8.8 \sigma$, respectively.

With a $\beta$ beating of $10 \%$, the BSC is reduced by about $0.4 \sigma$ and $0.5 \sigma$, respectively. Assuming a maximum $\beta$ beating of $5 \%$, which can be assumed from a rms $\beta$ beating of about $2 \%$ in the arcs, as demonstrated for the LHC [61], increases the BSC with already improved closed-orbit tolerances of $1 \mathrm{~mm}$ further to approximately $8.8 \sigma$ and $10.0 \sigma$. Hence, the $23 \times 90$ reaches the goal of $10 \sigma$ at $450 \mathrm{GeV}$ injection energy.

Another important contribution is found to arise from the mechanical aperture tolerances of $1 \mathrm{~mm}$ and results in about $0.9 \sigma$ and $1.0 \sigma$ BSC loss, respectively. An improvement by a factor of 2 of the mechanical tolerances would bring the BSC to approximately $9.2 \sigma$ and $10.5 \sigma$. Hence, the
$18 \times 90$ approaches to the goal of $10 \sigma$ at $450 \mathrm{GeV}$ injection energy.

Contrarily to the severe impact of the beforehand described parameters, which can lead to a significant BSC reduction, a parasitic dispersion of $14 \%$ reduces the BSC by about $0.1 \sigma$ and its possible reduction is therefore irrelevant for both HE-LHC FODO-cell layouts.

The analyses presented here suggest that the most efficient approach to reach the goal of $10 \sigma$ at $450 \mathrm{GeV}$ injection energy, thus avoiding increasing it, entails a revision of the closed-orbit and mechanical tolerances.

\section{Optimized layout of dispersion suppressors and lattices}

The structure of the DS is based on that of the LHC ring. The irregular part is made of eight main dipoles and three $4.5 \mathrm{~m}$-long individually powered quadrupoles (Q8-Q10), followed by a drift space of about $12 \mathrm{~m}$.

Particle tracking studies and energy-deposition simulations revealed the need for DS collimators (TCLDs), similar to those to be installed in HL-LHC, also for the FCC-hh [62,63], and for the HE-LHC [54,64-66]. The possibility of including two TCLDs was studied for each DS of the main experiments and collimation HE-LHC IRs, where one TCLD demands an additional space of $1.5 \mathrm{~m}$, located around Q8 and Q10. These additional TCLDs increase the length of the DS and hence the geometry offset with respect to the tunnel. Both irregular DS options are shown in Fig. 15. Two TCLDs are installed in all DS neighboring odd IRs to prove that the geometry offset can be kept small, although the length of these DSs is increased by $3 \mathrm{~m}$ compared to DSs without TCLDs.
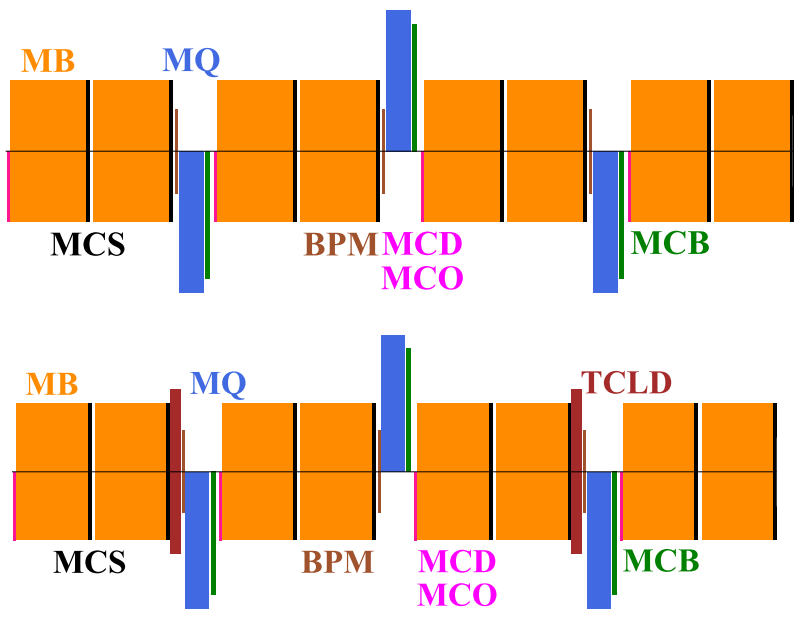

FIG. 15. Irregular part of the dispersion suppressor, right of the interaction region, consisting of eight main dipoles (MBs), each equipped with a sextupole spool piece corrector (MCS), three quadrupoles (MQ), three orbit correctors (MCB), three octupole (MCO) and decapole correctors (MCD) and three beam position monitors (BPMs), without (top) and with (bottom) collimators (TCLD). 
The regular DS lattice is identical to the first arc FODO cell, where, however, the first two arc main quadrupoles are powered individually. Periodic optics is therefore established after the first FODO cell. Due to peaks of $\beta$ function in the DS, some aperture bottlenecks appear there, with a BSC value of about $6 \sigma$ and $7 \sigma$ for the $18 \times 90$ and the $23 \times 90$ optics, respectively, at $450 \mathrm{GeV}$. The aperture restrictions can be mitigated using the strategies outlined in the previous section and by applying a refined optics rematching.

Optimized arc and DS lattice lead to a geometry offset with respect to LEP, comparable to that of LHC as seen in Fig. 16. A peak-to-peak transverse offset of 8.7 or $4.6 \mathrm{~cm}$ for the $18 \times 90$ or the $23 \times 90$ lattice, respectively, is found, where peaks are located in the DS. Due to the installed TCLDs, this offset is about $1.7 \mathrm{~cm}$ greater in DS neighboring odd interaction regions: without TCLDs, the peak-topeak offset decreases to 7.0 or $3.0 \mathrm{~cm}$, respectively. Moreover, due to TCLDs in the DS, interaction regions have an increased offset of about $5 \mathrm{~mm}$, which is, however, assumed to be negligible.

After geometry optimization, the ring optics is matched for injection and collision energy for both beams. Specially designed interaction regions are integrated in IR1, IR3, IR4, IR5, IR6 and IR7 [67-70], whereas in IR2 and IR8, lattice and optics are taken from LHC [1]. At injection energy, the arc trim quadrupoles are used to control the tune and set its fractional part to the LHC value of $(0.28,0.31)$.

High-luminosity experiments in IR1 and IR5 make use of a $\beta_{x, y}^{*}$ of $45 \mathrm{~cm}$. To match the fractional part of the working point at collision energy, i.e., $(0.31,0.32)$, the tune is controlled by means of the independently powered quadrupoles in IR4 and the main quadrupoles in the arcs $2-3,3-4,6-7$ and $7-8$, generating a small deviation of the FODO-cell phase advance, while preserving $90^{\circ}$ per cell in

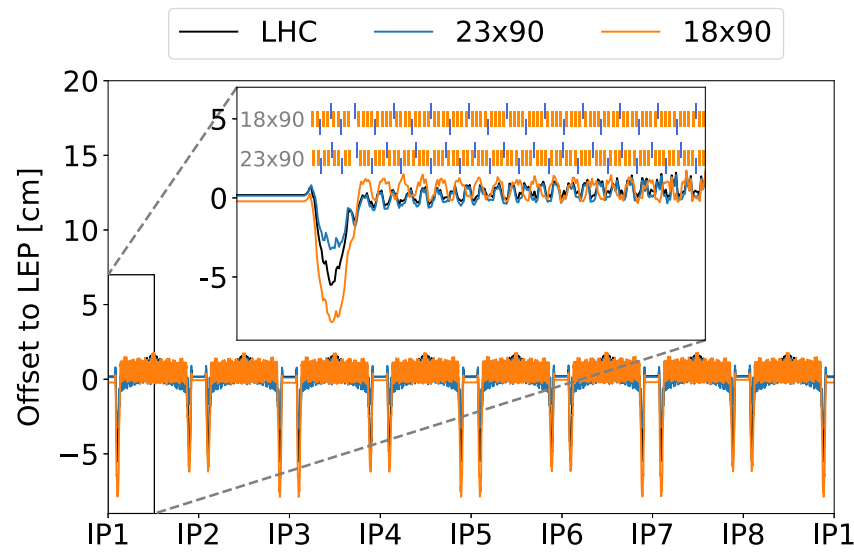

FIG. 16. Geometry offset of HE-LHC options and LHC with respect to LEP. Only the dispersion suppressors neighboring odd interaction regions include two TCLDs, as shown in Fig. 15 (bottom). the arcs neighboring the main experiments, to leave open the possibility to use ATS optics in future collision optics and to implement spurious dispersion correction schemes, similar to what is implemented in LHC.

The natural chromaticity of about -160 or -205 for the $18 \times 90$ or $23 \times 90$, respectively, at collision energy and -70 or -85 at injection energy, is corrected by the two families of chromatic sextupoles to +2 in both transverse planes.

The $18 \times 90$ lattice features a $66 \%$ higher momentum compaction factor than the $23 \times 90$, leading to lower transition energy, impacting longitudinal parameters as summarized in [71]. The optimized ring parameters for both options are listed in Table IV.

A half-crossing angle of $165 \mu \mathrm{rad}$, vertically or horizontally, for IR1 and IR5, respectively is deemed necessary, based on the results of beam-beam simulations, so to achieve a beam-beam long-range separation of at least $15.8 \sigma[11]$.

Spurious dispersion of 53 or $51 \mathrm{~cm}$, respectively for the $18 \times 90$ and the $23 \times 90$ lattices, is generated by the crossing bumps. Without appropriate correction, the spurious dispersion wave propagates through the lattice affecting negatively BSC, dynamic aperture, luminosity, and the functionalities of the IRs. Orbit bumps induced in arcs adjacent to the main experiments, generated by corrector dipoles acting on the corresponding crossing plane, lead to off-centered beams in quadrupoles and sextupoles, which, thanks to feed-down effects, can effectively control spurious dispersion over the whole arc [35].

To avoid linear-coupling effects generated by vertically off-centered particles passing through sextupoles, phase advances between the arc sextupoles and the IP should be equal to $n \times \frac{\pi}{2}$, where $n \in \mathbb{N}$. Although in the $23 \times 90$ optics this phase-advance constraint is not exactly fulfilled, still, the deviation is small enough to induce negligible skew quadrupole terms in the MAD-X model. Figure 17 shows the optics beating before spurious dispersion correction using orbit bumps for the $18 \times 90$ lattice. The induced orbit bumps compensate efficiently spurious dispersion within one arc, as shown in Fig. 18.

TABLE IV. Lattice parameters of the HE-LHC baseline options.

\begin{tabular}{lcc}
\hline \hline Parameter & $18 \times 90$ & $23 \times 90$ \\
\hline$\beta^{*}[\mathrm{~cm}]$ & \multicolumn{2}{c}{45} \\
Half crossing angle $[\mu \mathrm{rad}]$ & \multicolumn{2}{c}{165} \\
$\begin{array}{l}\text { Momentum compaction } \\
\quad \text { actor } \alpha_{\mathrm{c}}\left[10^{-4}\right]\end{array}$ & 5.8 & 3.5 \\
Transition energy $\gamma_{\mathrm{t}}[\mathrm{GeV}]$ & 41.5 & 53.8 \\
Natural chromaticity at injection & -70 & -85 \\
Natural chromaticity at collision & -162 & -205 \\
Tune injection $(\mathrm{H} / \mathrm{V})$ & $50.28 / 49.31$ & $61.28 / 58.31$ \\
Tune collision $(\mathrm{H} / \mathrm{V})$ & $50.31 / 49.32$ & $61.31 / 58.32$ \\
\hline \hline
\end{tabular}




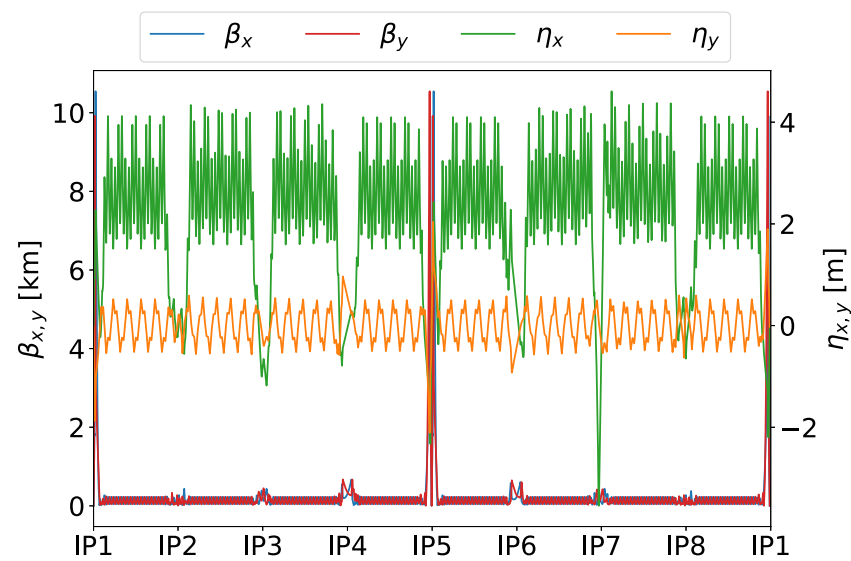

FIG. 17. Optics functions before spurious dispersion correction, arising from a crossing angle for the $18 \times 90$ lattice without dispersion suppressor collimators.
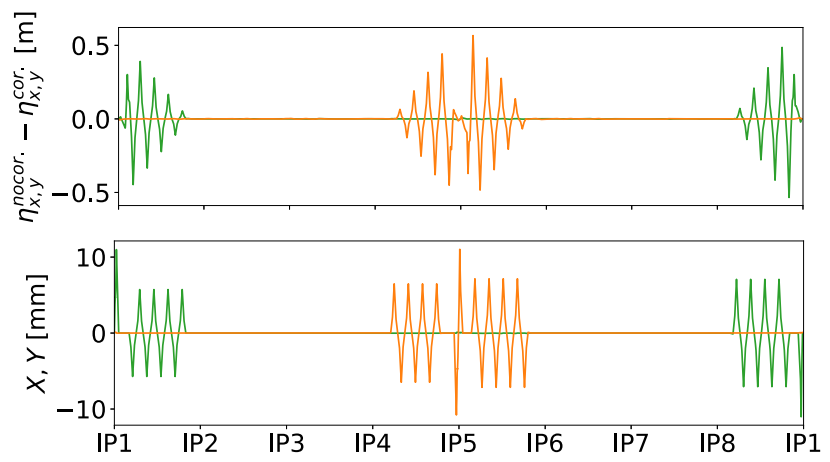

FIG. 18. Orbit bumps (bottom) and the resulting compensation of spurious dispersion (top) in the horizontal (green) and vertical (orange) plane for the $18 \times 90$ lattice without dispersion suppressor collimators. The compensation of spurious dispersion is displayed as the absolute difference between an optics without crossing angles, and an optics with crossing angles and spurious dispersion correction using orbit bumps.

It is worthwhile mentioning that the generation of the orbit bumps to correct the spurious dispersion has an adverse effect on the BSC. Therefore, a trade-off should be found between the aperture loss due to the orbit bumps and the harmful effect of the spurious dispersion, which also impacts on the ring aperture, but also on other items. Such a trade-off can be easily found by limiting the amplitude of the orbit bumps to what is strictly needed to reduce, not fully compensate, the spurious dispersion. Alternatively, further optimization of the nominal crossingangle value could be carried out, which would also reduce the value of the generated spurious dispersion and hence, in turns the amplitude of the needed orbit bump.

Lastly, the impact of the strong octupoles, used to control collective instabilities, on the feed-down due to these orbit bumps will need to be explored in further studies.

\section{HE-LHC FODO cells with $60^{\circ}$ phase advance}

Several $90^{\circ}$ [51] and $60^{\circ}$ [18] FODO-cell phase-advance designs have been studied and compared. Using $60^{\circ}$ phase advance together with designs that fulfill

$$
N_{\mathrm{c}} \mu_{x, y}=2 \pi k
$$

where $N_{\mathrm{c}}$ refers to the number of FODO cells per arc and $k \in \mathbb{N}$, have been found to suppress second-order effects from sextupoles and higher-order nonlinearities from magnet imperfections [18]. Another disadvantage of a $90^{\circ}$ phase-advance design is that it demands about $40 \%$ more integrated gradient than a $60^{\circ}$ one. Therefore, an alternative $60^{\circ}$ optics, based on the existing HE-LHC baseline designs with 18 and 23 FODO cells per arc, would imply the possibility to use shorter main quadrupoles, freeing space to increase the dipole filling factor and, hence, the energy reach. A $18 \times 60$ and a $23 \times 60$ arc optics is therefore analyzed while keeping the same cell lengths and conceptual lattice structure, as already shown in Fig. 12. FODO-cell parameters for these alternative optics are given in Table V.

For both options, dipole filling factor is increased and results in $82 \%$ and $79 \%$, leading to beam energies of 13.79 and $13.20 \mathrm{TeV}$, respectively, i.e., a gain of about $2 \%$ with respect to the corresponding $90^{\circ}$ optics counterparts, assuming an unchanged DS configuration. The values of the optical functions are increased in the lower phase advance FODO cells, which reduces the BSC by about $2.7 \sigma$ and $1.3 \sigma$, respectively for the 18 and the 23 FODO cells design. Strategies to reach the target BSC would hence need to be reviewed and optimized for the lower phase advance optics.

It has to be noted that implications on longitudinal dynamics, correction schemes, and dynamic aperture are not considered and would need to be explored in further studies in order to complete the assessment of these alternative optical configurations.

TABLE V. Parameters of the HE-LHC design options with $60^{\circ}$ phase advance.

\begin{tabular}{lcc}
\hline \hline Parameter & $18 \times 60$ & $23 \times 60$ \\
\hline Cell length [m] & 137.19 & 106.90 \\
Dipoles per cell & 8 & 6 \\
Dipole length [m] & 14.11 & 14.03 \\
Filling factor [\%] & 82 & 79 \\
Quadrupole length [m] & 2.0 & 2.3 \\
Quadrupole strength [Tm & -1 \\
$\beta_{\min } / \beta_{\max }$ in cell $[\mathrm{m}]$ & 340 & 356 \\
$D_{\min } / D_{\max }$ in cell $[\mathrm{m}]$ & $80 / 235$ & $63 / 182$ \\
Beam energy at $16 \mathrm{~T}[\mathrm{TeV}]$ & $4.0 / 6.7$ & $2.5 / 4.1$ \\
Field for $13.5 \mathrm{TeV}[\mathrm{T}]$ & 13.79 & 13.20 \\
BSC at $450 \mathrm{GeV}$ in arc $[\sigma]$ & 15.66 & 16.36 \\
\hline \hline
\end{tabular}




\section{LHC OPTICS WITH $60^{\circ}$ FODO-CELL PHASE ADVANCE}

To test the special HE-LHC optics with beam, a $60^{\circ} \mathrm{LHC}$ optics has been designed respecting all strength constraints discussed in Sec. III, based on the 2021 LHC lattice and optics configuration [72]. All operational aspects have been preserved, opening the possibility for testing this optics during LHC run 3 with a single, low-intensity bunch consisting of $10^{10}$ protons.

More than that, testing a $60^{\circ}$ optics in the LHC also enables interesting complementary studies, which will allow new insights on magnet imperfections, sextupole spool piece misalignments [73], linear and nonlinear coupling $[74,75]$ and the corresponding correction techniques. Indeed, understanding optics with different FODOcell phase advance for the same lattice will not only help to constrain error sources, but will, in addition, probe optics flexibility, which is a challenge for future machines such as the FCC-ee [7], which features a $60^{\circ}$ and a $90^{\circ}$ optics.

Lattice parameters of a $60^{\circ} \mathrm{LHC}$ injection optics together with nominal $90^{\circ}$ injection optics are given in Table VI, where aperture parameters described in Sec. III are used.

Optics functions in FODO cells with $60^{\circ}$ increase with respect to a $90^{\circ}$ optics due to weaker quadrupole strengths, as shown in Fig. 19.

This leads to new boundary conditions between the arcs, the DS, and the IRs. A rematching of the whole ring optics is therefore unavoidable. For the injection optics, $\beta_{x, y}^{*}$ at IP $1 / 2 / 5 / 8$ is $11 / 10 / 11 / 10 \mathrm{~m}$, which is unchanged with respect to the nominal 2021 LHC injection optics. Phaseadvance constraints required for beam injection in IR2 and IR8 for beam 1 and beam 2, respectively, are preserved. Collimation optics in IR3 and IR7 are kept unchanged between Q6 on the left and right side of the IP. In IR4, the optics in between the rf cavities remain unchanged. For the beam dump in IR6, the $\beta$ functions remain unchanged, however the horizontal dispersion is about $1 \mathrm{~m}$ smaller in the $60^{\circ}$ optics. Sextupole strengths for chromaticity

TABLE VI. $60^{\circ}$ and nominal $90^{\circ} \mathrm{LHC}$ injection optics parameters at $450 \mathrm{GeV}$, using aperture parameters described in Sec. III. The maximum beam energy considers only MQ gradient limits.

\begin{tabular}{|c|c|c|}
\hline Parameter & $60^{\circ} \mathrm{LHC}$ & $90^{\circ} \mathrm{LHC}$ \\
\hline Maximum beam energy $[\mathrm{TeV}]$ & 12.3 & 7.5 \\
\hline$\beta_{\min } / \beta_{\max }[\mathrm{m}]$ & $63 / 182$ & $32 / 177$ \\
\hline$D_{\min } / D_{\max }[\mathrm{m}]$ & $2.5 / 4.1$ & $1.1 / 2.2$ \\
\hline$\alpha_{\mathrm{c}}\left[10^{-4}\right]$ & 6.9 & 3.5 \\
\hline$\gamma_{\mathrm{t}}[\mathrm{GeV}]$ & 40.0 & 53.6 \\
\hline Natural chromaticity & -60 & -83 \\
\hline Corrected chromaticity & 2 & 2 \\
\hline MS strength $\left[\mathrm{Tm}^{-2}\right]$ & 56 & 142 \\
\hline Tune injection $(\mathrm{H} / \mathrm{V})$ & $45.28 / 44.31$ & $62.28 / 60.31$ \\
\hline $\mathrm{BSC}$ at $450 \mathrm{GeV}$ in $\operatorname{arc}[\sigma]$ & 13.2 & 13.4 \\
\hline
\end{tabular}

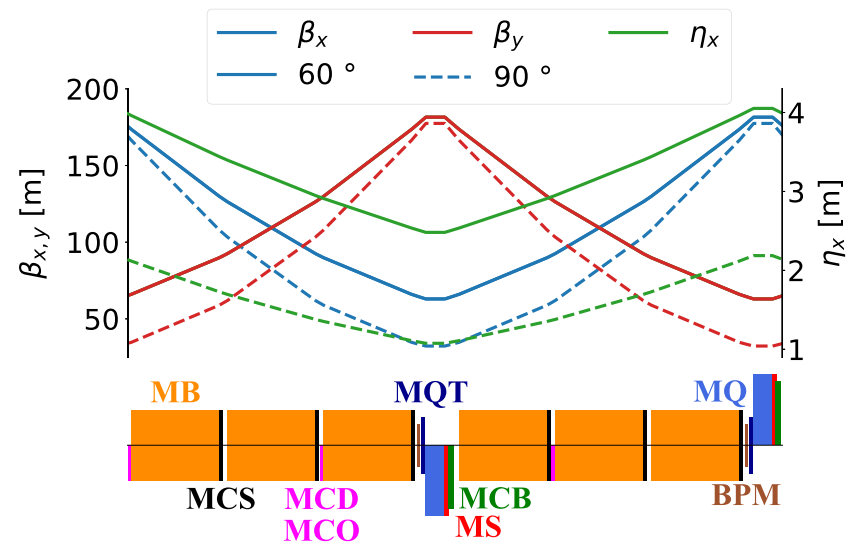

FIG. 19. LHC FODO cell together with the $\beta$ functions and the dispersion function $\left(\eta_{x}\right)$ for a $60^{\circ}$ (solid line) and a $90^{\circ}$ (dashed line) design.

correction are reduced by a factor 3 due to lower natural chromaticity and larger dispersion.

Weaker quadrupoles increase the optics functions and hence the beam size, which leads to smaller available aperture, and smaller BSC. However, using HL-LHC aperture parameters, a sufficient BSC of about $13 \sigma$ is found in the regular arc structure at $450 \mathrm{GeV}$. Bottlenecks arise in the DS due to local optics peaks, where minima of $8.16 \sigma$ are found in the DS located left of the main IRs, but this is assumed to be sufficient for a pilot test bunch.

The lower phase advance leads to lower integer tunes, while the fractional part of the tunes is matched identical to LHC, i.e., $Q_{x} / Q_{y}=45.31 / 44.32$ for collision. To match the working point at the nominal injection values, i.e., $Q_{x} / Q_{y}=45.28 / 44.31$, the families of the arc trim quadrupoles are used. Contrarily to the $90^{\circ}$ optics, a $\beta$ beating is introduced in the $60^{\circ}$ optics when using the trimming quadrupoles. Changing the tunes by 0.01 introduces a rms $\beta$ beating of $0.55 \%$ using all families, but it can be reduced
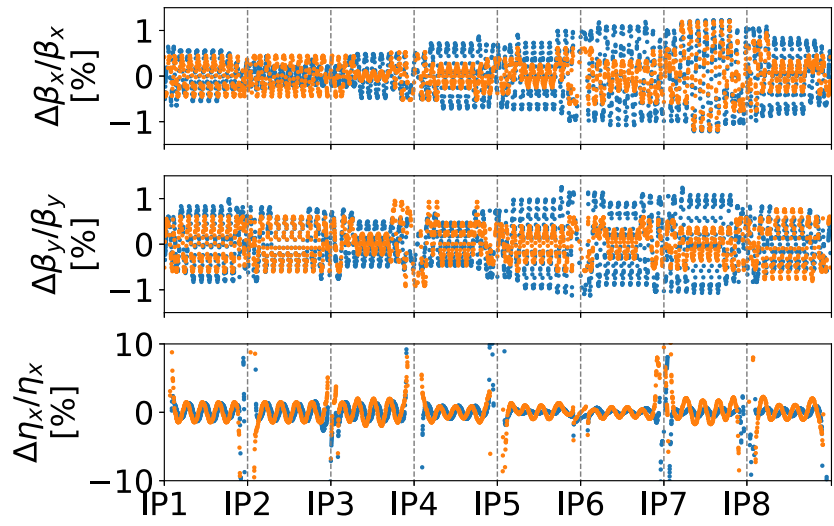

FIG. 20. Introduced $\beta$ beating and dispersion $(\eta)$ beating using 16 MQT families (blue) and using eight MQT families (orange) for trimming tunes by 0.01 with respect to nominal collision tunes. 
by using only half of the families, leading to a rms $\beta$ beating of $0.40 \%$, as shown in Fig. 20. In any case, such a $\beta$ beating is not deemed to be an obstacle for the beam tests.

Linear coupling is corrected by means of dedicated skew quadrupoles located in the arcs and in the IRs [76]. In spite of the different FODO-cell phase advance and reduced integer tune split, coupling knobs can be defined, which introduce a maximum vertical dispersion below $2 \mathrm{~mm}$.

Finally, it is worth noting that the momentum compaction $\alpha_{\mathrm{c}}$ is increased by a factor 2 for the $60^{\circ}$ optics, leading to a $\sqrt{2}$ lower transition energy $\gamma_{\mathrm{t}}$. This implies the additional benefit of a smaller momentum spread at injection, after capture and filamentation, and a decrease of the longitudinal emittance required for beam stability during ramp and collisions (Sec. 2.4.9 of [11] and also [77,78]).

\section{CONCLUSIONS}

Design options for various possible energy upgrades of the HL-LHC have been studied and discussed in detail in this paper. The assumption is that these circular accelerators should be installed in the existing LHC tunnel and ancillary infrastructure, hence imposing strong geometry constraints onto the layout, at the same time not hampering the goal of increasing beam energy above $7 \mathrm{TeV}$.

Partial replacement of the existing LHC main dipoles by stronger ones, assuming the feasibility of $16 \mathrm{~T}$ dipoles with a design derived from that assumed for the FCC-hh, reach beam energies of 9.53 or $11.50 \mathrm{TeV}$ by replacing $1 / 3$ or $2 / 3$ of arc main dipoles, respectively. The dispersion suppressors impose the installation of new main dipoles to compensate for the change of bending strengths in the arcs so to keep the geometry offset with respect to the current LHC small. All new dipoles must be bent to reach a BSC of at least $10 \sigma$, assuming HL-LHC parameters aperture computation and FCC-hh beam screen at $450 \mathrm{GeV}$. Better aperture for the upgraded rings can be achieved by optimizing the design of the beam screen of the new dipoles, thus deviating from the FCC-hh cross section.

It should be noted that the main arc quadrupoles are limited to $8.6 \mathrm{TeV}$ with a $90^{\circ}$ phase-advance optics. Nevertheless, it has been shown that a mitigation measure can be found by designing an optics with $60^{\circ}$ phase advance and for this option the strength limit of the main quadrupoles would be lifted to $12.3 \mathrm{TeV}$, thus allowing a major part of HL-LHC elements to be reused. However, and perhaps even more importantly, several other magnets should still be replaced, namely separation dipoles and insertion quadrupoles, in addition to dedicated engineering efforts to adapt other components to the increased beam energy, and to address the interfaces between new and old magnets, including their vacuum chambers.

Existing HE-LHC options, representing a full energy upgrade of the HL-LHC, have been reviewed in depth and fully optimized. A very complete set of solutions has been provided and discussed in detail. Great emphasis has been put into optimizing both baseline lattices, resulting in acceptable deviations with respect to the LHC and LEP geometry. Thorough optimization work has made the layout of the dispersion suppressors better and compatible with the option of adding collimators in all DS neighboring IRs, housing the main experiments and the collimation system, while keeping the geometry offset with respect to LEP comparable to LHC for both baseline options.

The issue of available aperture at injection energy has been considered in detail as this has deep implications on the feasibility of a given optics and ring layout, going up to modifications of the SPS as injector. A number of solutions to reach the minimum BSC target of $10 \sigma$ have been proposed to overcome the difficulties found in past versions of the HE-LHC design: An optimal beam screen geometry has been worked out and is ready for further studies aiming at its engineering optimization. This would enable keeping the SPS as injector of the HE-LHC or to perform a limited upgrade of this accelerator. Note that the use of combinedfunction dipoles has been explored as a means to improve the overall aperture of the ring and it has been shown that the BSC target can be reached by including an additional $b_{2}$ component of $\pm 515 \times 10^{-4}$ or $\pm 500 \times$ $10^{-4}$ (at $R_{\text {ref }}=16.7 \mathrm{~mm}$ ) in the main dipoles, respectively, for the $18 \times 90$ and the $23 \times 90$ option. Furthermore, a careful study of the impact of the values assumed for the parameters used in the aperture computations has been carried out. Indeed, to achieve the target BSC it could also be envisaged to assume an improved correction of the closed orbit as well as a better mechanical alignment. It is also worth noting that the achieved level of optics quality in the LHC, if assumed in the aperture computations, would have a sizable beneficial impact on the BSC estimate. As an example, the target BSC value of $10 \sigma$ can be achieved for the $23 \times 90$ optics if the residual closed-orbit error is set to $1 \mathrm{~mm}$ and the residual $\beta$ beating is $2 \%$, which corresponds to the measured $\beta$ beating in the LHC.

The optics design has been brought to maturity by developing and implementing the most advanced features to ensure optimal performance. For example, it includes schemes to correct spurious dispersion based on orbit bumps [35]. In addition, as an alternative to the baseline optics featuring a $90^{\circ}$ phase advance in the arc FODO cells, arc designs with $60^{\circ}$ have been developed. These alternative optics require lower gradients for the arc quadrupoles, thus allowing for a higher dipole filling factor, and thereby even further increasing the energy of the baseline design.

Lastly, an offspring of these studies is the development of a $60^{\circ}$ FODO-cell phase-advance optics that can be used in the present LHC for dedicated beam dynamics studies in run 3. In addition, to probing optics solutions for future accelerators, measuring and analyzing a $60^{\circ}$ LHC optics will offer new insights into the LHC beam dynamics. They will also help us better understand the sources of LHC 
optics errors, through comparing the beam behavior for two different optical configurations.

\section{ACKNOWLEDGMENTS}

This work was supported by the FCC study and the HLLHC project, as well as by the Austrian doctoral student program. We acknowledge enlightening collaborations and numerous helpful discussions with Y. Nosochkov and Y. Cai of SLAC, and with D. Zhou of KEK, concerning the optimum HE-LHC FODO-cell structure. Y. Nosochkov and Y. Cai first proposed a $60^{\circ}$ optics for the HE-LHC. We are also thankful to E. Shaposhnikova and T. Persson for fruitful discussions on the longitudinal parameters and on the beam-stay clear calculations in MAD-X respectively. Finally, we would like to express our warm thanks to J. Jowett for providing us with a detailed historical recollection of the evolution of the LHC arc-cell design. This work was supported, in part, by the European Commission under the HORIZON2020 Integrating Activity project ARIES, grant agreement 73087.

[1] O. Brüning et al., LHC design report, CERN, Geneva, CERN Yellow Reports, Monographs, 2004.

[2] High-luminosity Large Hadron Collider (HL-LHC): Technical design report V. 0.1, edited by G. Apollinari, A. I. Béjar, O. Brüning, P. Fessia, M. Lamont, L. Rossi, and L. Tavian, Report No. CERN-2017-007-M, 2017.

[3] FCC study, https://fcc.web.cern.ch/.

[4] M. Benedikt, European Circular Energy Frontier Collider Study, H2020-INFRADEV-1-2014-1, Report No. 654305, 2014.

[5] M. Benedikt et al., FCC-hh: The hadron collider, Eur. Phys. J. Special Topics 228, 755 (2019).

[6] M. Benedikt, A. Blondel, P. Janot, M. Mangano, and F. Zimmermann, Future circular colliders succeeding the LHC, Nat. Phys. 16, 402 (2020).

[7] M. Benedikt et al., FCC-ee: The Lepton Collider, Eur. Phys. J. Special Topics 228, 261 (2019).

[8] CERN, LEP design report, Report No. CERN-LEP-84-01, CERN, Geneva, 1984.

[9] The CEPC Study Group, CEPC Conceptual Design Report: Volume 1-Accelerator, arXiv:1809.00285.

[10] Proceedings, EuCARD-AccNet-EuroLumi workshop: The high-energy Large Hadron Collider (HE-LHC10), Villa Bighi, Malta, Republic of Malta, 2010, edited by E. Todesco and F. Zimmermann, CERN Yellow Report No. CERN-2011-003, 2011.

[11] F. Zimmermann et al., HE-LHC: The high-energy Large Hadron Collider, Eur. Phys. J. Special Topics 228, 1109 (2019).

[12] F. Bordry, M. Benedikt, O. Brüning, J. Jowett, L. Rossi, D. Schulte, S. Stapnes, and F. Zimmermann, Machine parameters and projected luminosity performance of proposed future colliders at CERN, CERN, Geneva, Report No. CERN-ACC-2018-0037, 2018.
[13] Report on the physics at the HL-LHC, and perspectives for the HE-LHC, edited by A. Dainese, M. Mangano, A. B. Meyer, A. Nisati, G. Salam, and M. A. Vesterinen, CERN Yellow Report, CERN, Geneva, Report No. CERN-2019007, 2019.

[14] LHC luminosity and energy upgrade: A feasibility study, edited by F. Ruggiero et al., CERN, Geneva, Report No. LHC-Project-Report-626, 2002.

[15] S. Fartoukh, M. Giovannozzi, D. Missiaen, E. Todesco, and F. Zimmermann, Considerations on a partial energy upgrade of the LHC, CERN, Geneva, Report No. CERNACC-2017-096, 2017.

[16] O. Brüning et al., LHC full energy exploitation study: Operation at ultimate energy of $7.5 \mathrm{TeV}$, CERN, Geneva, Report No. CERN-ACC-2019-0015, 2019.

[17] O. Brüning et al., LHC full energy exploitation study: Upgrade for operation beyond ultimate energy of 7.5 TeV, CERN, Geneva, Report No. CERN-ACC-2020-0015, 2020.

[18] Y. Nosochkov, Y. Cai, M. Crouch, M. Giovannozzi, M. Hofer, J. Keintzel, T. Risselada, E. Todesco, R. Tomás, D. Zhou, F. Zimmermann, and L. van Riesen-Haupt, Optimized arc optics for the HE-LHC, in Proceedings of the 9th International Particle Accelerator Conference (IPAC'18), Vancouver, BC, Canada (JACoW, Geneva, Switzerland, 2018), MOPMF067.

[19] A. A. Garren and D. E. Johnson, Modifications to the SSC lattice, in Proceedings of the 1987 Particle Accelerator Conference, Washington, DC (IEEE, New York, 1987).

[20] J. D. Jackson et al., Superconducting super collider conceptual design, Report No. SSC-SR-2020, 1986.

[21] A. A. Garren and D. E. Johnson, Status of the SSC lattice design, in Proceedings of the First European Particle Accelerator Conference EPAC88, Rome (World Scientific, Singapore, 1989).

[22] A. M. Asner et al., Large hadron collider in the LEP tunnel: A feasibility study of possible options, Report No. CERNDIR-TECH-84-01, CERN, Geneva, Switzerland, 1984.

[23] J. P. Koutchouk, W. Scandale, A. Verdier, and M. Bassetti, Optimization of the LHC lattice and chromaticity, in Proceedings of the 1987 Particle Accelerator Conference (Ref. [19]).

[24] The Large Hadron Collider in the LEP tunnel, edited by G. Brianti and K. Hübner, Report No. CERN-87-05, CERN, Geneva, Switzerland, 1987.

[25] W. Scandale, The lattice of the CERN-LHC for high luminosity pp collisions, Reports No. CERN-SPS-88-6AMS and No. CERN-LHC-Note-68, CERN, Geneva, Switzerland, 1988.

[26] W. Scandale, B. Jeanneret, J.-P. Koutchouk, X. Luo, F. Mot, R. Ostojic, T. Risselada, C. Rufer, T. Taylor, T. Trenkler, and $S$. Weisz, The lattice of the CERN Large Hadron Collider, in Proceedings of the Particle Accelerator Conference, Dallas, TX, 1995 (IEEE, New York, 1995).

[27] J. Coello, R. Tomás, L. Fiscarelli, D. Gamba, and M. Martino, Impact of flux jumps in future colliders, Phys. Rev. Accel. Beams 23, 011001 (2020).

[28] R. Bruce, R. W. Assmann, and S. Redaelli, Calculations of safe collimator settings and $\beta^{*}$ at the CERN Large Hadron Collider, Phys. Rev. ST Accel. Beams 18, 061001 (2015). 
[29] S.D. Fartoukh, Chromatic coupling induced by skew sextupolar field errors in the LHC main dipoles and its correction, LHC-Project-Report-278, 1999.

[30] E. H. Maclean, R. Tomás, F. S. Carlier, M. SolfaroliCamillocci, J. W. Dilly, J. C. de Portugal, E. Fol, K. Fuchsberger, A. G.-T. Valdivieso, M. Giovannozzi, M. Hofer, L. Malina, T. H. B. Persson, P. K. Skowronski, and A. Wegscheider, New approach to LHC optics commissioning for the nonlinear era, Phys. Rev. Accel. Beams 22, 061004 (2019).

[31] T. H. B. Persson and R. Tomás, Optimizing chromatic coupling measurements in the LHC, at the International Particle Accelerator Conference 2016, Busan, Korea (JACoW, Geneva, Switzerland, 2016), TUPMW036.

[32] S. Y. Lee, Accelerator Physics, 3rd ed. (World Scientific, Singapore, 2012).

[33] http://usmdp.lbl.gov/.

[34] G. Velev, G. Ambrosio, E. Barzi, V. V. Kashikhin, S. Krave, V. Lombardo, I. Novitski, S. Stoynev, D. Turrioni, $\mathrm{X}$. Xu, and A. V. Zlobin, Fermilab superconducting $\mathrm{Nb}_{3} \mathrm{Sn}$ high field magnet R\&D program, in Proceedings of the 10th International Particle Accelerator Conference (IPAC'19), Melbourne, Australia (JACoW, Geneva, Switzerland, 2019), THPTS099, pp. 4338-4341.

[35] S. Fartoukh, Achromatic telescopic squeezing scheme and application to the LHC and its luminosity upgrade, Phys. Rev. ST Accel. Beams 16, 111002 (2013).

[36] R. Burgmer, M. Durante, H.-U. Klein, D. Krischel, M. Peyrot, J.-M. Rifflet, B. Schellong, K.-M. Schirm, P. Schmidt, F. Simon, T. Stephani, T. Tortschanoff, and W. Venturini-Delsolaro, Performance of the first LHC main quadrupoles made in industry, in Proceedings of the 2003 Particle Accelerator Conference, Portland, OR (IEEE, New York, 2003), WPAE007.

[37] L. van Riesen-Haupt, Advanced accelerator interaction region optics for LHC operation and future hadron colliders, Ph.D. Thesis, University of Oxford, 2019.

[38] J.-B. Jeanneret and R. Ostojic, Geometrical acceptance in LHC version 5.0, Report No. LHC-Project-Note-111, 1997.

[39] MAD-X, http://mad.web.cern.ch/mad/.

[40] J. B. Jeanneret, Geometrical tolerances for the qualification of LHC magnets, LHC Project Report No. 1007, CERN, 2006.

[41] R. Bruce, R. de Maria, S. Fartoukh, M. Giovannozzi, S. Radelli, R. Tomás, and J. Wenninger, Parameters for HLLHC aperture calculations, Report No. CERN-ACC-20140044, 2014, http://cds.cern.ch/record/1697805?ln=en.

[42] R. Bruce, C. Bracco, R. De Maria, M. Giovannozzi, S. Redaelli, R. Tomás, F. Velotti, and J. Wenninger, Parameters for aperture calculations at injection for HL-LHC, Report No. CERN-ACC-2016-0328, 2016, https://cds.cern .ch/record/2237427? ln=en.

[43] R. Bruce, C. Bracco, R. De Maria, M. Giovannozzi, S. Redaelli, R. Tomás, F. Velotti, and J. Wenninger, Updated parameters for HL-LHC aperture calculations for proton beams, Report No. CERN-ACC-2017-0051, 2017, https:// cds.cern.ch/record/2274330? In=en.

[44] O. Brüning and S. Fartoukh, Field quality specification for the LHC main dipole magnets, CERN, Geneva, Report No. CERN-LHC-Project-Report-501, 2001.
[45] R. W. Assmann, The final collimation system for the LHC, in Proceedings of the 10th European Particle Accelerator Conference, Edinburgh, Scotland, 2006 (EPS-AG, Edinburgh, Scotland, 2006), p. 986.

[46] R. Bruce et al., Simulations and measurements of beam loss patterns at the CERN Large Hadron Collider, Phys. Rev. ST Accel. Beams 17, 081004 (2014).

[47] G. Valentino, G. Baud, R. Bruce, M. Gasior, A. Mereghetti, D. Mirarchi, J. Olexa, S. Redaelli, B. Salvachua, A. Valloni, and J. Wenninger, Final implementation, commissioning, and performance of embedded collimator beam position monitors in the Large Hadron Collider, Phys. Rev. Accel. Beams 20, 081002 (2017).

[48] R. Bruce, C. Bracco, R. De Maria, M. Giovannozzi, A. Mereghetti, D. Mirarchi, S. Redaelli, E. Quaranta, and B. Salvachua, Reaching record-low $\beta^{*}$ at the CERN Large Hadron Collider using a novel scheme of collimator settings and optics, Nucl. Instrum. Methods Phys. Res., Sect. A 848, 19 (2017).

[49] I. Bellafont, M. Morrone, L. Mether, J. Fernndez, R. Kersevan, C. Garion, V. Baglin, P. Chiggiato, and F. Prez, Design of the future circular hadron collider beam vacuum chamber, Phys. Rev. Accel. Beams 23, 033201 (2020).

[50] T. Risselada (private communication).

[51] J. Keintzel, Arc cell options for the HE-LHC, https://cds .cern.ch/record/2624282.

[52] J. Keintzel, M. Crouch, M. Hofer, L. v. Riesen-Haupt, T. Risselada, R. Tomás, and F. Zimmermann, HE-LHC optics design options, at the 10th International Particle Accelerator Conference (IPAC'19), Melbourne, Australia (JACoW, Geneva, Switzerland, 2019), MOPMP026.

[53] M. Hofer, M. Giovannozzi, J. Keintzel, R. Tomás, F. Zimmermann, and L. van Riesen-Haupt, Dynamic aperture at injection for the HE-LHC, at the 10th International Particle Accelerator Conference (IPAC'19), Melbourne, Australia (JACoW, Geneva, Switzerland, 2019), MOPMP023.

[54] F. Zimmermann et al., Updated high-energy LHC design, at the 10th International Particle Accelerator Conference (IPAC'19), Melbourne, Australia (JACoW, Geneva, Switzerland, 2019), MOPMP037.

[55] R. Tomás, M. Benedikt, M. Hofer, J. Keintzel, and F. Zimmermann, Towards future colliders, Proc. Sci., LHCP2018 (2018) 268.

[56] R. Tomás, J. Keintzel, and S. Papadopoulou, Emittance growth from luminosity burn-off in future hadron colliders, Phys. Rev. Accel. Beams 23, 031002 (2020).

[57] J. Keintzel, Optics design and performance aspects of the HE-LHC, Report No. CERN-THESIS-2018-177, 2018.

[58] A. Verdier, Resonance free lattices for alternating gradient machines, Report No. CERN-SL-99-018-AP, 1999.

[59] CERN, The $300 \mathrm{GeV}$ programme, https://cds.cern.ch/ record/104068.

[60] T. Risselada, LHC dynamic aperture with a square beam screen cross section, Report No. SL-AP Note 91-21, 1991.

[61] T. Persson, F. Carlier, J. C. de Portugal, A. G.-T. Valdivieso, A. Langner, E. H. Maclean, L. Malina, P. Skowronski, B. Salvant, R. Tomás, and A. C. G. Bonilla, LHC optics commissioning: A journey towards 1\% optics control, Phys. Rev. Accel. Beams 20, 061002 (2017). 
[62] M. Fiascaris, R. Bruce, and S. Redaelli, A conceptual solution for a beam halo collimation system for the future circular hadron-hadron collider (FCC-hh), Nucl. Instrum. Methods Phys. Res., Sect. A 894, 96 (2018).

[63] R. Bruce, A. Abramov, A. Bertarelli, M. I. Besana, F. Carra, F. Cerutti, A. Faus-Golfe, M. Fiascaris, G. Gobbi, A. Krainer, A. Lechner, A. Mereghetti, D. Mirarchi, J. Molson, M. Pasquali, S. Redaelli, D. Schulte, M. Serluca, E. Skordis, and M. Varasteh, Collimation system studies for the FCC-hh, J. Phys. Conf. Ser. 1350, 012009 (2019).

[64] A. Abramov, R. Bruce, M. Crouch, N. Fuster-Martinez, A. Mereghetti, J. Molson, L. J. Nevay, and S. Redaelli, Collimation of heavy-ion beams in the HE-LHC, in Proceedings of the 10th International Particle Accelerator Conference (IPAC'19), Melbourne, Australia (JACoW, Geneva, Switzerland, 2019), MOPRB059, pp. 704-707.

[65] M. Varasteh and F. Cerutti, Update on energy deposition studies for HE-LHC and FCC-hh, FCC collimation design meeting \#23 (2019), https://indico.cern.ch/event/826277/.

[66] M. Varasteh, R. Bruce, F. Cerutti, M. Crouch, and F. Zimmermann, Impact of betatron collimation losses in the High Energy Large Hadron Collider, submitted to Phy. Rev. Accel. Beams, 2020.

[67] L. van Riesen-Haupt, J. L. Abelleira, M. Crouch, E. C. Alaniz, A. Seryi, D. Zhou, and F. Zimmermann, Experimental interaction region optics for the high energy LHC, J. Phys. Conf. Ser. 1067, 022002 (2018).

[68] L. van Riesen-Haupt, J. L. Abelleira, E. C. Alaniz, M. Hofer, P. M. Mirave, A. Seryi, D. Zhou, and F. Zimmermann, Optics for rf acceleration section for the high energy Large Hadron Collider, in Proceedings of the 9th International Particle Accelerator Conference (IPAC'18), Vancouver, Canada (JACoW, Geneva, Switzerland, 2018), pp. 345-347.
[69] W. Bartmann, M. J. Barnes, L. Ducimetire, B. Goddard, M. Hofer, T. Kramer, A. Lechner, E. Renner, A. S. Ull, V. Senaj, L. S. Stoel, and C. Wiesner, Injection and dump systems for a $13.5 \mathrm{TeV}$ hadron synchrotron HE-LHC, J. Phys. Conf. Ser. 1067, 052021 (2018).

[70] T. Risselada (private communication).

[71] E. Shaposhnikova, Longitudinal parameters, at the FCC week 2019, Brussels, Amsterdam, 2019, https://indico.cern .ch/event/727555/contributions/3439883/attachments/ 1868461/3073451/HE_ES.pdf.

[72] S. Fartoukh, LHC run 3 optics status, at the BE-ABP-HSS meeting, CERN, Geneva, 2019, https://indico.cern.ch/ event $/ 866609 /$.

[73] F. Hulphers, $\beta$-beating and amplitude detuning from corrector misalignments in the LHC and HL-LHC, Report No. CERN-STUDENTS-Note-2019-257, 2019.

[74] M. Hofer and R. Tomás, Effect of local linear coupling on linear and nonlinear observables in circular accelerators, Phys. Rev. Accel. Beams 23, 094001 (2020).

[75] E. H. Maclean, F. Carlier, M. Giovannozzi, T. H. B. Persson, and R. Tomás, Effect of linear coupling on nonlinear observables at the LHC, in Proceedings of the 8th International Particle Accelerator Conference (IPAC'17), Copenhagen, Denmark (JACoW, Geneva, Switzerland, 2017), WEPIK092, pp. 3151-3154.

[76] R. Tomás, Optimizing the global coupling knobs for the LHC, Report No. CERN-ATS-Note-2012-019, CERN, Geneva, 2014.

[77] E. Shaposhnikova (private communication).

[78] E. Shaposhnikova, Longitudinal beam parameters during acceleration in the LHC, Report No. LHC-PROJECTNOTE-242, CERN, Geneva, Switzerland, 2020. 